\title{
Extended Gray-Wyner System with Complementary Causal Side Information
}

\author{
Cheuk Ting Li and Abbas El Gamal \\ Department of Electrical Engineering, Stanford University \\ Email: ctli@stanford.edu, abbas@ee.stanford.edu
}

\begin{abstract}
We establish the rate region of an extended Gray-Wyner system for 2-DMS $(X, Y)$ with two additional decoders having complementary causal side information. This extension is interesting because in addition to the operationally significant extreme points of the Gray-Wyner rate region, which include Wyner's common information, Gács-Körner common information and information bottleneck, the rate region for the extended system also includes the Körner graph entropy, the privacy funnel and excess functional information, as well as three new quantities of potential interest, as extreme points. To simplify the investigation of the 5-dimensional rate region of the extended Gray-Wyner system, we establish an equivalence of this region to a 3-dimensional

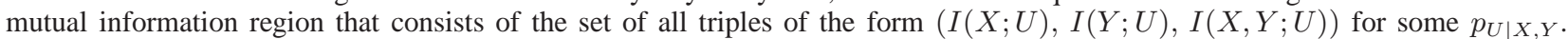
We further show that projections of this mutual information region yield the rate regions for many settings involving a 2-DMS, including lossless source coding with causal side information, distributed channel synthesis, and lossless source coding with a helper.
\end{abstract}

\section{Index Terms}

Gray-Wyner system, side information, complementary delivery, Körner graph entropy, privacy funnel.

\section{INTRODUCTION}

The lossless Gray-Wyner system [1] is a multi-terminal source coding setting for two discrete memoryless source (2-DMS) $(X, Y)$ with one encoder and two decoders. This setup draws some of its significance from providing operational interpretation for several information theoretic quantities of interest, namely Wyner's common information [2], the Gács-Körner common information [3], the necessary conditional entropy [4], and the information bottleneck [5].

In this paper, we consider an extension of the Gray-Wyner system (henceforth called the EGW system), which includes two new individual descriptions and two decoders with causal side information as depicted in Figure 1 The encoder maps sequences from a 2-DMS $(X, Y)$ into five indices $M_{i} \in\left[1: 2^{n R_{i}}\right], i=0, \ldots, 4$. Decoders 1 and 2 correspond to those of the Gray-Wyner system, that is, decoder 1 recovers $X^{n}$ from $\left(M_{0}, M_{1}\right)$ and decoder 2 recovers $Y^{n}$ from $\left(M_{0}, M_{2}\right)$. At time $i \in[1: n]$, decoder 3 recovers $X_{i}$ causally from $\left(M_{0}, M_{3}, Y^{i}\right)$ and decoder 4 similarly recovers $Y_{i}$ causally from $\left(M_{0}, M_{4}, X^{i}\right)$. Note that decoders 3 and 4 correspond to those of the complementary delivery setup studied in [6], [7] with causal (instead of noncausal) side information and with two additional private indices $M_{3}$ and $M_{4}$. This extended Gray-Wyner system setup is lossless, that is, the decoders recover their respective source sequences with probability of error that vanishes as $n$ approaches infinity. The rate region $\mathscr{R}$ of the EGW system is defined in the usual way as the closure of the set of achievable rate tuples $\left(R_{0}, R_{1}, R_{2}, R_{3}, R_{4}\right)$.

The first contribution of this paper is to establish the rate region of the EGW system. Moreover, to simplify the study of this rate region and its extreme points, we show that it is equivalent to the 3-dimensional mutual information region for $(X, Y)$ defined as

$$
\mathscr{I}_{X Y}=\bigcup_{p_{U \mid X Y}}\{(I(X ; U), I(Y ; U), I(X, Y ; U))\} \subseteq \mathbb{R}^{3}
$$

in the sense that we can express $\mathscr{R}$ using $\mathscr{I}$ and vice versa. As a consequence and of particular interest, the extreme points of the rate region $\mathscr{R}$ (and its equivalent mutual information region $\mathscr{I}_{X Y}$ ) for the EGW system include, in addition to the aforementioned extreme points of the Gray-Wyner system, the Körner graph entropy [8], privacy funnel [9] and excess functional information [10], as well as three new quantities with interesting operational meaning, which we refer to as the maximal interaction information, the asymmetric private interaction information, and the symmetric private interaction information. These extreme points can be cast as maximizations of the interaction information [11] $I(X ; Y \mid U)-I(X ; Y)$ under various constraints. They can be considered as distances from extreme dependency, as they are equal to zero only under certain conditions of extreme dependency. In addition to providing operational interpretations to these information theoretic quantities, projections of the mutual information region yield the rate regions for many settings involving a 2-DMS, including lossless source coding with causal side information [12], distributed channel synthesis [13], [14], and lossless source coding with a helper [15], [16], [17].

A related extension of lossy Gray-Wyner system with two decoders with causal side information was studied by Timo and Vellambi [18]. If we only consider decoders 3 and 4 in EGW, then it can be considered as a special case of their setting 


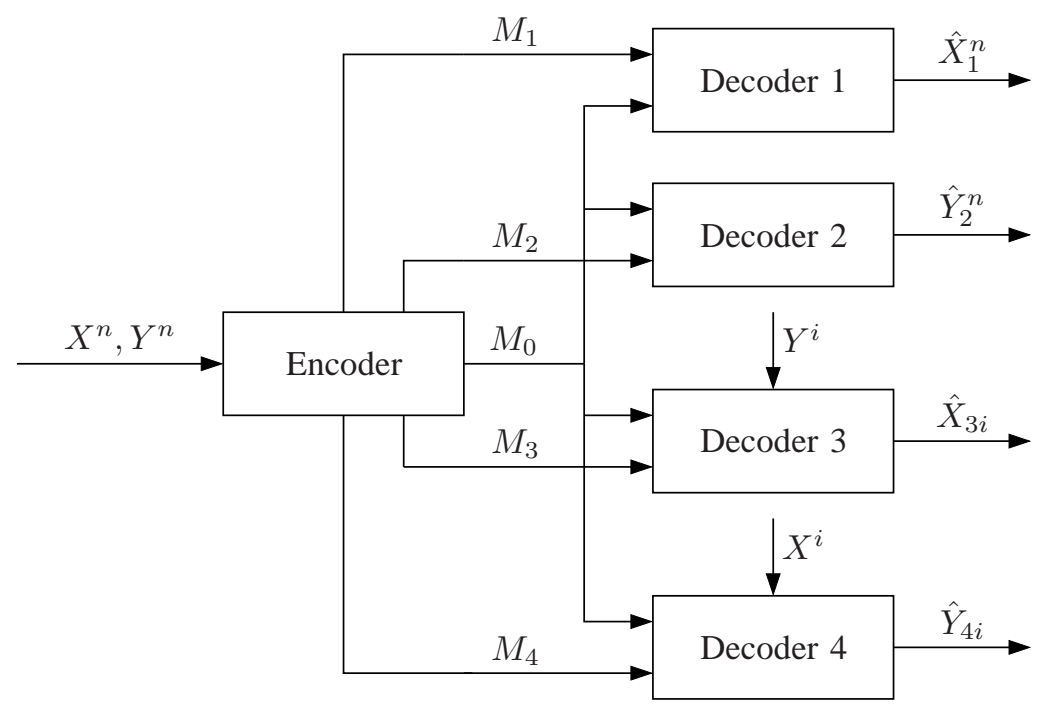

Figure 1. Extended Gray-Wyner system.

(where the side information does not need to be complementary). Other related source coding setups to the EGW can be found in [19], [12], [20], [21], [22]. A related 3-dimensional region, called the region of tension, was investigated by Prabhakaran and Prabhakaran [23], [24]. We show that this region can be obtained from the mutual information region, but the other direction does not hold in general.

In the following section, we establish the rate region of the EGW system, relate it to the mutual information region, and show that the region of the original Gray-Wyner system and the region of tension can be obtained from the mutual information region. In Section III, we study the extreme points of the mutual information region. In Section IV we establish the rate region for the same setup as the EGW system but with noncausal instead of causal side information at decoders 3 and 4 . We show that the rate region of the noncausal EGW can be expressed in terms of the Gray-Wyner region, hence it does not contain as many interesting extreme points as the causal EGW. Moreover, we show that this region is equivalent to the closure of the limit of the mutual information region for $\left(X^{n}, Y^{n}\right)$ as $n$ approaches infinity.

\section{A. Notation}

Throughout this paper, we assume that log is base 2 and the entropy $H$ is in bits. We use the notation: $X_{a}^{b}=\left(X_{a}, \ldots, X_{b}\right)$, $X^{n}=X_{1}^{n}$ and $[a: b]=[a, b] \cap \mathbb{Z}$.

For discrete $X$, we write the probability mass function as $p_{X}$. For $A \subseteq \mathbb{R}^{n}$, we write the closure of $A$ as $\operatorname{cl}(A)$ and the convex hull as $\operatorname{conv}(A)$. We write the support function as

$$
\psi_{A}(b)=\sup \left\{a^{T} b: a \in A\right\} .
$$

We write the one-sided directional derivative of the support function as

$$
\psi_{A}^{\prime}(b ; c)=\lim _{t \rightarrow 0^{+}} \frac{1}{t}\left(\psi_{A}(b+t c)-\psi_{A}(b)\right) .
$$

Note that if $A$ is compact and convex, then

$$
\psi_{A}^{\prime}(b ; c)=\max \left\{d^{T} c: d \in \underset{a \in A}{\arg \max } a^{T} b\right\} .
$$

\section{RATE REGION OF EGW AND THE MUTUAL INFORMATION REGION}

The rate region of the EGW system is given in the following.

Theorem 1. The rate region the EGW system $\mathscr{R}$ is the set of rate tuples $\left(R_{0}, R_{1}, R_{2}, R_{3}, R_{4}\right)$ such that

$$
\begin{aligned}
& R_{0} \geq I(X, Y ; U), \\
& R_{1} \geq H(X \mid U), \\
& R_{2} \geq H(Y \mid U), \\
& R_{3} \geq H(X \mid Y, U), \\
& R_{4} \geq H(Y \mid X, U)
\end{aligned}
$$


for some $p_{U \mid X Y}$, where $|\mathcal{U}| \leq|\mathcal{X}| \cdot|\mathcal{Y}|+2$.

Note that if we ignore decoders 3 and 4, i.e., let $R_{3}, R_{4}$ be sufficiently large, then this region reduces to the Gray-Wyner region.

Proof: The converse proof is quite straightforward and is given in Appendix $\mathrm{A}$ for completion. We now prove the achievability.

Codebook generation. Fix $p_{U \mid X Y}$ and randomly and independently generate $2^{n R_{0}}$ sequences $u^{n}\left(m_{0}\right), m_{0} \in\left[1: 2^{n R_{0}}\right]$, each according to $\prod_{i=1}^{n} p_{U}\left(u_{i}\right)$. Given $u^{n}\left(m_{0}\right)$, assign indices $m_{1} \in\left[1: 2^{n R_{1}}\right], m_{2} \in\left[1: 2^{n R_{2}}\right]$ to the sequences in the conditional typical sets $\mathcal{T}_{\epsilon}^{(n)}\left(X \mid u^{n}\left(m_{0}\right)\right)$ and $\mathcal{T}_{\epsilon}^{(n)}\left(Y \mid u^{n}\left(m_{0}\right)\right)$, respectively. For each $y \in \mathcal{Y}, u \in \mathcal{U}$, assign indices $m_{3, y, u} \in\left[1: 2^{n R_{3, y, u} p_{Y U}(y, u)}\right]$ to the sequences in $\mathcal{T}_{\epsilon}^{n(1+\epsilon) p_{Y U}(y, u)}(X \mid y, u)$, where $\sum_{y, u} R_{3, y, u} p_{Y U}(y, u) \leq R_{3}$. Define $m_{4, x, u}$ similarly.

Encoding. To encode the sequence $x^{n}, y^{n}$, find $m_{0}$ such that $\left(u^{n}\left(m_{0}\right), x^{n}, y^{n}\right) \in \mathcal{T}_{\epsilon}^{(n)}$ is jointly typical, and find indices $m_{1}, m_{2}$ of $x^{n}, y^{n}$ in $\mathcal{T}_{\epsilon}^{(n)}\left(X \mid u^{n}\left(m_{0}\right)\right)$ and $\mathcal{T}_{\epsilon}^{(n)}\left(Y \mid u^{n}\left(m_{0}\right)\right)$ given $u^{n}\left(m_{0}\right)$. For each $x, y$, let $x_{y, u}^{n}$ be the subsequence of $x^{n}$ where $x_{i}$ is included if and only if $y_{i}=y$ and $u_{i}\left(m_{0}\right)=u$. Note that since $\left(u^{n}\left(m_{0}\right), y^{n}\right) \in \mathcal{T}_{\epsilon}^{(n)}$, the length of $x_{y, u}^{n}$ is not greater than $n(1+\epsilon) p_{Y U}(y, u)$. We then find an index $m_{3, y, u}$ of $\hat{x}_{y, u}^{n(1+\epsilon) p_{Y U}(y, u)} \in \mathcal{T}_{\epsilon}^{n(1+\epsilon) p_{Y U}(y, u)}(X \mid y, u)$ such that $x_{y, u}^{n}$ is a prefix of $\hat{x}_{y, u}^{n(1+\epsilon) p_{Y U}(y, u)}$, and output $m_{3}$ as the concatenation of $m_{3, y, u}$ for all $y, u$. Similar for $m_{4}$.

Decoding. Decoder 1 outputs the sequence corresponding to the index $m_{1}$ in $\mathcal{T}_{\epsilon}^{(n)}\left(X \mid u^{n}\left(m_{0}\right)\right)$. Decoder 2 performs similarly using $\left(m_{0}, m_{2}\right)$. Decoder 3 , upon observing $y_{i}$, finds the sequence $\hat{x}_{y_{i}, u_{i}\left(m_{0}\right)}^{n(1+\epsilon) p_{Y U}\left(y_{i}, u_{i}\left(m_{0}\right)\right)}$ at the index $m_{3, y_{i}, u_{i}\left(m_{0}\right)}$ in $\mathcal{T}_{\epsilon}^{n(1+\epsilon) p_{Y U}\left(y_{i}, u_{i}\left(m_{0}\right)\right)}\left(X \mid y_{i}, u_{i}\left(m_{0}\right)\right)$, and output the next symbol in the sequence that is not previously used. Decoder 4 performs similarly using $\left(m_{0}, m_{4}\right)$.

Analysis of the probability of error. By the covering lemma, the probability that there does not exist $m_{0}$ such that $\left(u^{n}\left(m_{0}\right), x^{n}, y^{n}\right) \in$ $\mathcal{T}_{\epsilon}^{(n)}$ tends to 0 if $R_{0}>I(X, Y ; U)$. Also $\left|\mathcal{T}_{\epsilon}^{(n)}\left(X \mid u^{n}\left(m_{0}\right)\right)\right| \leq 2^{n R_{1}}$ for large $n$ if $R_{1}>H(X \mid U)+\delta(\epsilon)$ (similar for $\left.R_{2}>H(Y \mid U)+\delta(\epsilon)\right)$. Note that $\left(u^{n}\left(m_{0}\right), x^{n}, y^{n}\right) \in \mathcal{T}_{\epsilon}^{(n)}$ implies

$$
\begin{aligned}
\frac{\left|\left\{i: x_{i}=x, y_{i}=y, u_{i}\left(m_{0}\right)=u\right\}\right|}{n(1+\epsilon) p_{Y U}(y, u)} & \leq \frac{(1+\epsilon) p_{X Y U}(x, y, u)}{(1+\epsilon) p_{Y U}(y, u)} \\
& \leq p_{X \mid Y U}(x \mid y, u)
\end{aligned}
$$

for all $(y, u)$. Hence there exists $\hat{x}_{y, u}^{n(1+\epsilon) p_{Y U}(y, u)} \in \mathcal{T}_{\epsilon}^{n(1+\epsilon) p_{Y U}(y, u)}(X \mid y, u)$ such that $x_{y, u}^{n}$ is a prefix of $\hat{x}_{y, u}^{n(1+\epsilon) p_{Y U}(y, u)}$. And $\left|\mathcal{T}_{\epsilon}^{n(1+\epsilon) p_{Y U}(y, u)}(X \mid y, u)\right| \leq 2^{n R_{3, y, u} p_{Y U}(y, u)}$ for large $n$ if $R_{3, y, u}>(1+\epsilon) H(X \mid Y=y, U=u)+\delta(\epsilon)$. Hence we can assign suitable $R_{3, y, u}$ for each $y, u$ if $R_{3}>(1+\epsilon) H(X \mid Y, U)+\delta(\epsilon)$.

Although $\mathscr{R}$ is 5-dimensional, the bounds on the rates can be expressed in terms of three quantities: $I(X ; U), I(Y ; U)$ and $I(X, Y ; U)$ together with other constant quantities that involve only the given $(X, Y)$. This leads to the following equivalence of $\mathscr{R}$ to the mutual information region $\mathscr{I}_{X Y}$ defined in (1). We denote the components of a vector $v \in \mathscr{I}_{X Y}$ by $v=$ $\left(v_{X}, v_{Y}, v_{X Y}\right)$.

Proposition 1. The rate region for the EGW system can be expressed as

$$
\mathscr{R}=\bigcup_{v \in \mathscr{I}_{X Y}}\left\{\left(v_{X Y}, H(X)-v_{X}, H(Y)-v_{Y}, H(X \mid Y)-v_{X Y}+v_{Y}, H(Y \mid X)-v_{X Y}+v_{X}\right)\right\}+[0, \infty)^{5},
$$

where the last “+” denotes the Minkowski sum. Moreover, the mutual information region for $(X, Y)$ can be expressed as

$$
\mathscr{I}_{X Y}=\left\{v \in \mathbb{R}^{3}:\left(v_{X Y}, H(X)-v_{X}, H(Y)-v_{Y}, H(X \mid Y)-v_{X Y}+v_{Y}, H(Y \mid X)-v_{X Y}+v_{X}\right) \in \mathscr{R}\right\} .
$$

Proof: Note that (2) follows from the definitions of $\mathscr{R}$ and $\mathscr{I}_{X Y}$. We now prove (3). The $\subseteq$ direction follows from (2). For the $\supseteq$ direction, let $v \in \mathbb{R}^{3}$ satisfy

$$
\left(v_{X Y}, H(X)-v_{X}, H(Y)-v_{Y}, H(X \mid Y)-v_{X Y}+v_{Y}, H(Y \mid X)-v_{X Y}+v_{X}\right) \in \mathscr{R} .
$$

Then by Theorem 1, there exists $U$ such that

$$
\begin{gathered}
v_{X Y} \geq I(X, Y ; U), \\
H(X)-v_{X} \geq H(X \mid U), \\
H(Y)-v_{Y} \geq H(Y \mid U), \\
H(X \mid Y)-v_{X Y}+v_{Y} \geq H(X \mid Y, U), \\
H(Y \mid X)-v_{X Y}+v_{X} \geq H(Y \mid X, U) .
\end{gathered}
$$


Adding (4) and (8), we have $v_{X} \geq I(X ; U)$. Combining this with (5), we have $v_{X}=I(X ; U)$. Similarly $v_{Y}=I(Y ; U)$. Substituting this into (7), we have $v_{X Y} \leq I(X, Y ; U)$. Combining this with (4), we have $v_{X Y}=I(X, Y ; U)$. Hence $v \in \mathscr{I}_{X Y}$.

In the following we list several properties of $\mathscr{I}_{X Y}$.

Proposition 2. The mutual information region $\mathscr{I}_{X Y}$ satisfies:

1) Compactness and convexity. $\mathscr{I}_{X Y}$ is compact and convex.

2) Outer bound. $\mathscr{I}_{X Y} \subseteq \mathscr{I}_{X Y}^{\mathrm{o}}$, where $\mathscr{I}_{X Y}^{\mathrm{o}}$ is the set of $v$ such that

$$
\begin{gathered}
v_{X}, v_{Y} \geq 0 \\
v_{X}+v_{Y}-v_{X Y} \leq I(X ; Y), \\
0 \leq v_{X Y}-v_{Y} \leq H(X \mid Y), \\
0 \leq v_{X Y}-v_{X} \leq H(Y \mid X)
\end{gathered}
$$

3) Inner bound. $\mathscr{I}_{X Y} \supseteq \mathscr{I}_{X Y}^{\mathrm{i}}$, where $\mathscr{I}_{X Y}^{\mathrm{i}}$ is the convex hull of the points $(0,0,0),(H(X), I(X ; Y), H(X))$, $(I(X ; Y), H(Y), H(Y)),(H(X), H(Y), H(X, Y)),(H(X \mid Y), H(Y \mid X), H(X \mid Y)+H(Y \mid X))$.

Moreover, there exists $0 \leq \epsilon_{1}, \epsilon_{2} \leq \log I(X ; Y)+4$ such that

$$
\left(0, H(Y \mid X)-\epsilon_{1}, H(Y \mid X)\right),\left(H(X \mid Y)-\epsilon_{2}, 0, H(X \mid Y)\right) \in \mathscr{I}_{X Y} .
$$

4) Superadditivity. If $\left(X_{1}, Y_{1}\right)$ is independent of $\left(X_{2}, Y_{2}\right)$, then

$$
\mathscr{I}_{X_{1}, Y_{1}}+\mathscr{I}_{X_{2}, Y_{2}} \subseteq \mathscr{I}_{\left(X_{1}, X_{2}\right),\left(Y_{1}, Y_{2}\right)},
$$

where + denotes the Minkowski sum. As a result, if $\left(X_{i}, Y_{i}\right) \sim p_{X Y}$ i.i.d. for $i=1, \ldots, n, \mathscr{I}_{X Y} \subseteq(1 / n) \mathscr{I}_{X}, Y^{n}$.

5) Data processing. If $X_{2}-X_{1}-Y_{1}-Y_{2}$ forms a Markov chain, then for any $v \in \mathscr{I}_{X_{1}, Y_{1}}$, there exists $w \in \mathscr{I}_{X_{2}, Y_{2}}$ such that $w_{X} \leq v_{X}, w_{Y} \leq v_{Y}, w_{X Y} \leq v_{X Y}$

$$
I\left(X_{2} ; Y_{2}\right)-w_{X}-w_{Y}+w_{X Y} \leq I\left(X_{1} ; Y_{1}\right)-v_{X}-v_{Y}+v_{X Y}
$$

6) Cardinality bound.

$$
\mathscr{I}_{X Y}=\bigcup_{p_{U \mid X Y}:|\mathcal{U}| \leq|\mathcal{X}| \cdot|\mathcal{Y}|+2}\{(I(X ; U), I(Y ; U), I(X, Y ; U))\} .
$$

7) Relation to Gray-Wyner region and region of tension. The Gray-Wyner region can be obtained from $\mathscr{I}_{X Y}$ as

$$
\begin{aligned}
\mathscr{R}_{\mathrm{GW}} & =\bigcup_{p_{U \mid X Y}}\{(I(X, Y ; U), H(X \mid U), H(Y \mid U))\}+[0, \infty)^{3} \\
& =\bigcup_{v \in \mathscr{I}_{X Y}}\left\{\left(v_{X Y}, H(X)-v_{X}, H(Y)-v_{Y}\right)\right\}+[0, \infty)^{3} .
\end{aligned}
$$

The region of tension can be obtained from $\mathscr{I}_{X Y}$ as

$$
\begin{aligned}
\mathfrak{T} & =\bigcup_{p_{U \mid X Y}}\{(I(Y ; U \mid X), I(X ; U \mid Y), I(X ; Y \mid U))\}+[0, \infty)^{3} \\
& =\bigcup_{v \in \mathscr{I}_{X Y}}\left\{\left(v_{X Y}-v_{X}, v_{X Y}-v_{Y}, I(X ; Y)-v_{X}-v_{Y}+v_{X Y}\right)\right\}+[0, \infty)^{3} .
\end{aligned}
$$

The proof of this proposition is given in Appendix $B$

\section{Extreme Points of the Mutual Information Region}

Many interesting information theoretic quantities can be expressed as optimizations over $\mathscr{I}_{X Y}$ (and $\mathscr{R}$ ). Since $\mathscr{I}_{X Y}$ is convex and compact, some of these quantities can be represented in terms of the support function $\psi_{\mathscr{I}_{X Y}}(x)$ and its one-sided directional derivative, which provides a representation of those quantities using at most 6 coordinates. To avoid conflicts and for consistency, we use different notation for some of these quantities from the original literature . We use semicolons, e.g., $G(X ; Y)$, for symmetric quantities, and arrows, e.g., $G(X \rightarrow Y)$, for asymmetric quantities.

Figures 2, 3 illustrate the mutual information region $\mathscr{I}_{X Y}$ and its extreme points, and Table 1 lists the extreme points and their corresponding optimization problems and support function representations.

We first consider the extreme points of $\mathscr{I}_{X Y}$ that correspond to previously known quantities.

Wyner's common information [2]

$$
J(X ; Y)=\min _{X-U-Y} I(X, Y ; U)
$$




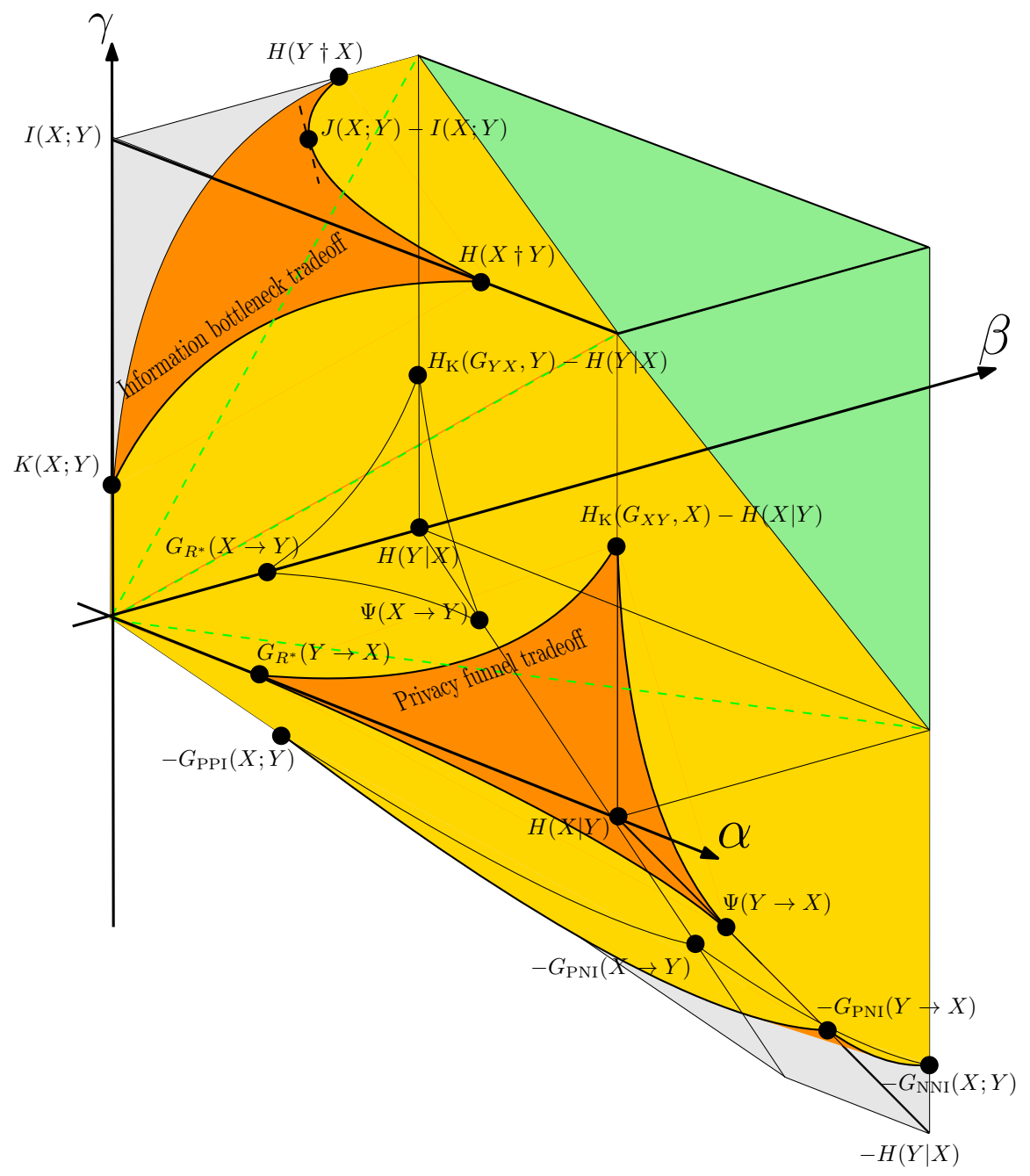

Figure 2. Illustration of $\mathscr{I}_{X Y}$ (yellow), $\mathscr{I}_{X Y}^{\mathrm{i}}$ (green) and $\mathscr{I}_{X Y}^{\mathrm{o}}$ (grey) defined in Proposition 2 The axes are $\alpha=I(X ; U \mid Y)=v_{X Y}-v_{Y}$, $\beta=I(Y ; U \mid X)=v_{X Y}-v_{X}$ and $\gamma=v_{X}+v_{Y}-v_{X Y}$, i.e., the mutual information $I(X ; Y ; U)$. Without loss of generality, we assume $H(X) \geq H(Y)$. Note that the original Gray-Wyner region and the region of tension correspond to the upper-left corner.

can be expressed as

$$
\begin{aligned}
J(X ; Y) & =\min \left\{v_{X Y}: v \in \mathscr{I}_{X Y}, v_{X}+v_{Y}-v_{X Y}=I(X ; Y)\right\} \\
& =\min \left\{R_{0}: R_{0}^{4} \in \mathscr{R}, R_{0}+R_{1}+R_{2}=H(X, Y)\right\} \\
& =-\psi_{\mathscr{I}_{X Y}}^{\prime}(1,1,-1 ; 0,0,-1) .
\end{aligned}
$$

\section{Gács-Körner common information [3], [25]}

$$
K(X ; Y)=\max _{U: H(U \mid X)=H(U \mid Y)=0} H(U)=\max _{U: X-Y-U, U-X-Y} I(X, Y ; U)
$$

can be expressed as

$$
\begin{aligned}
K(X ; Y) & =\max \left\{v_{X Y}: v \in \mathscr{I}_{X Y}, v_{X}=v_{Y}=v_{X Y}\right\} \\
& =\max \left\{R_{0}: R_{0}^{4} \in \mathscr{R}, R_{0}+R_{1}=H(X), R_{0}+R_{2}=H(Y)\right\} \\
& =\psi_{\mathscr{I}_{X Y}}^{\prime}(1,1,-2 ; 0,0,1) .
\end{aligned}
$$

Körner graph entropy [8], [26]. Let $G_{X Y}$ be a graph with a set of vertices $\mathcal{X}$ and edges between confusable symbols upon observing $Y$, i.e., there is an edge $\left(x_{1}, x_{2}\right)$ if $p\left(x_{1}, y\right), p\left(x_{2}, y\right)>0$ for some $y$. The Körner graph entropy

$$
H_{\mathrm{K}}\left(G_{X Y}, X\right)=\min _{U: U-X-Y, H(X \mid Y, U)=0} I(X ; U)
$$



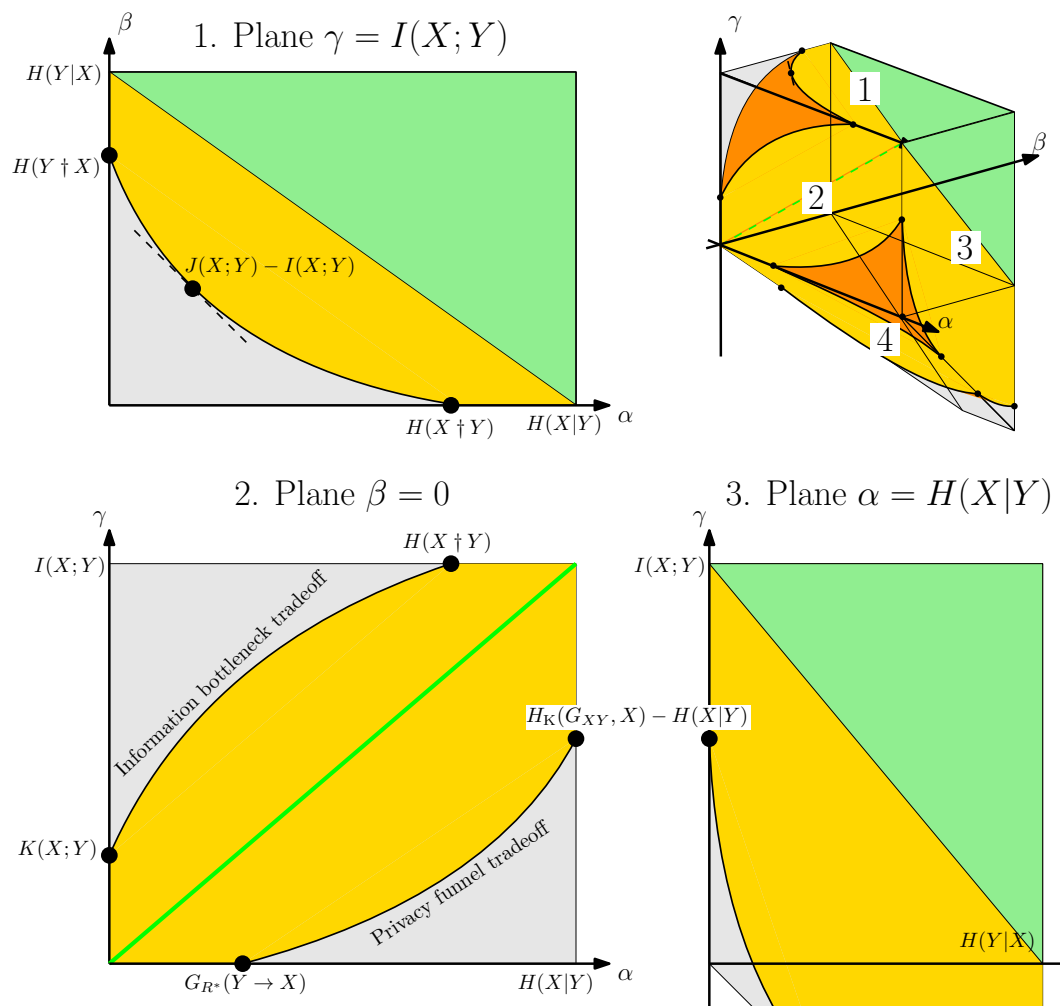

3. Plane $\alpha=H(X \mid Y)$

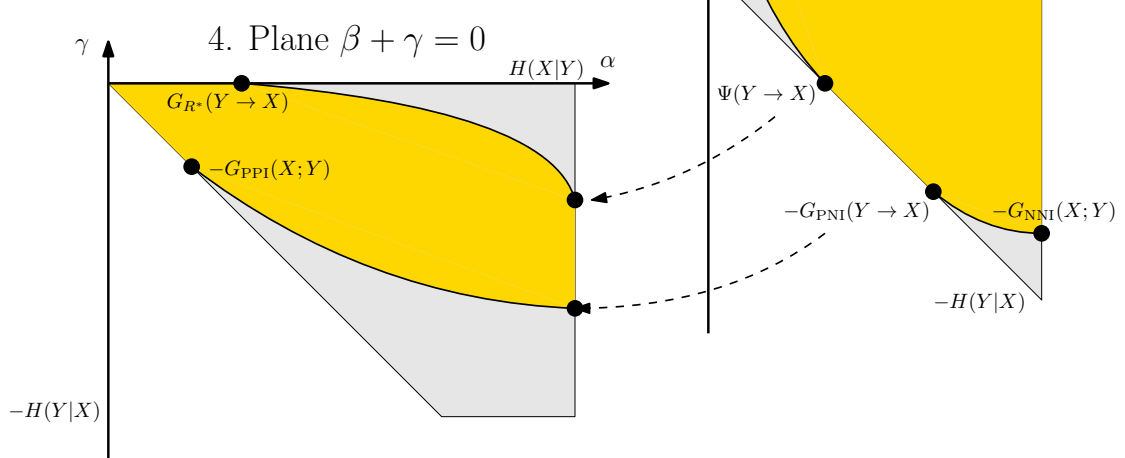

Figure 3. Illustration of $\mathscr{I}_{X Y}$ (yellow), $\mathscr{I}_{X Y}^{\mathrm{i}}$ (green) and $\mathscr{I}_{X Y}^{\mathrm{o}}$ (grey) restricted to different planes. The axes are $\alpha=I(X ; U \mid Y)=v_{X Y}-v_{Y}$, $\beta=I(Y ; U \mid X)=v_{X Y}-v_{X}$ and $\gamma=v_{X}+v_{Y}-v_{X Y}$. We assume $H(X) \geq H(Y)$.

can be expressed as

$$
\begin{aligned}
H_{\mathrm{K}}\left(G_{X Y}, X\right) & =\min \left\{v_{X}: v \in \mathscr{I}_{X Y}, v_{X}=v_{X Y}, v_{X Y}-v_{Y}=H(X \mid Y)\right\} \\
& =\min \left\{R_{0}: R_{0}^{4} \in \mathscr{R}, R_{0}+R_{1}=H(X), R_{3}=0\right\} \\
& =-\psi_{\mathscr{I}_{X Y}}^{\prime}(1,-1,0 ;-1,0,0) .
\end{aligned}
$$

In the Gray-Wyner system with causal complementary side information, $H_{\mathrm{K}}\left(G_{X Y}, X\right)$ corresponds to the setting with only decoders 1,3 and $M_{3}=\emptyset$, and we restrict the sum rate $R_{0}+R_{1}=H(X)$. This is in line with the lossless source coding setting with causal side information [12], where the optimal rate is also given by $H_{\mathrm{K}}\left(G_{X Y}, X\right)$. An intuitive reason of this equality is that $R_{0}+R_{1}=H(X)$ and the recovery requirement of decoder 1 forces $M_{0}$ and $M_{1}$ to contain negligible information outside $X^{n}$, hence the setting is similar to the case in which the encoder has access only to $X^{n}$. This corresponds to lossless source coding with causal side information setting.

Necessary conditional entropy [4] (also see $H(Y \searrow X \mid X)$ in [27], $G(Y \rightarrow X)$ in [28], private information in [29] and [30])

$$
H(Y \dagger X)=\min _{U: H(U \mid Y)=0, X-U-Y} H(U \mid X)=\min _{U: X-Y-U, X-U-Y} I(Y ; U)-I(X ; Y)
$$


can be expressed as

\section{Information bottleneck [5]}

$$
\begin{aligned}
H(Y \dagger X) & =\min \left\{v_{X Y}: v \in \mathscr{I}_{X Y}, v_{Y}=v_{X Y}, v_{X}=I(X ; Y)\right\}-I(X ; Y) \\
& =\min \left\{R_{0}: R_{0}^{4} \in \mathscr{R}, R_{0}+R_{2}=H(Y), R_{1}=H(X \mid Y)\right\} \\
& =-\psi_{\mathscr{I}_{X Y}}^{\prime}(1,2,-2 ; 1,0,-1) .
\end{aligned}
$$

can be expressed as

$$
G_{\mathrm{IB}}(t, X \rightarrow Y)=\min _{U: X-Y-U, I(X ; U) \geq t} I(Y ; U)
$$

$$
\begin{aligned}
G_{\mathrm{IB}}(t, X \rightarrow Y) & =\min \left\{v_{Y}: v \in \mathscr{I}_{X Y}, v_{Y}=v_{X Y}, v_{X} \geq t\right\} \\
& =\min \left\{R_{0}: R_{0}^{4} \in \mathscr{R}, R_{0}+R_{2}=H(Y), R_{1} \leq H(X)-t\right\} .
\end{aligned}
$$

Note that the same tradeoff also appears in common randomness extraction on a 2-DMS with one-way communication [31], lossless source coding with a helper [15], [16], [17], and a quantity studied by Witsenhausen and Wyner [32]. It is shown in [33] that its slope is given by the chordal slope of the hypercontractivity of Markov operator [34]

$$
\begin{aligned}
s^{*}(Y \rightarrow X) & =\sup _{U: X-Y-U} \frac{I(X ; U)}{I(Y ; U)} \\
& =\sup \left\{v_{X} / v_{Y}: v \in \mathscr{I}_{X Y}, v_{Y}=v_{X Y}\right\} .
\end{aligned}
$$

Privacy funnel [9] (also see the rate-privacy function defined in [29])

$$
G_{\mathrm{PF}}(t, X \rightarrow Y)=\min _{U: X-Y-U, I(Y ; U) \geq t} I(X ; U)
$$

can be expressed as

$$
\begin{aligned}
G_{\mathrm{PF}}(t, X \rightarrow Y) & =\min \left\{v_{X}: v \in \mathscr{I}_{X Y}, v_{Y}=v_{X Y}, v_{Y} \geq t\right\} \\
& =\min \left\{R_{0}+R_{4}-H(Y \mid X): R_{0}^{4} \in \mathscr{R}, R_{0}+R_{2}=H(Y), R_{0} \geq t\right\} .
\end{aligned}
$$

In particular, the maximum $R$ for perfect privacy (written as $g_{0}(X ; Y)$ in [29], also see [35]) is

$$
\begin{aligned}
G_{R^{*}}(X \rightarrow Y) & =\max \left\{t \geq 0: G_{\mathrm{PF}}(t, X \rightarrow Y)=0\right\} \\
& =\max \left\{v_{Y}: v \in \mathscr{I}_{X Y}, v_{Y}=v_{X Y}, v_{X}=0\right\} \\
& =\max \left\{R_{0}: R_{0}^{4} \in \mathscr{R}, R_{0}+R_{2}=H(Y), R_{0}+R_{4}=H(Y \mid X)\right\} \\
& =\psi_{\mathscr{I}_{X Y}}^{\prime}(-1,1,-1 ; 0,1,0) .
\end{aligned}
$$

The optimal privacy-utility coefficient [35] is

$$
\begin{aligned}
v^{*}(X \rightarrow Y) & =\inf _{U: X-Y-U} \frac{I(X ; U)}{I(Y ; U)} \\
& =\inf \left\{v_{X} / v_{Y}: v \in \mathscr{I}_{X Y}, v_{Y}=v_{X Y}\right\} .
\end{aligned}
$$

Excess functional information [10]

$$
\Psi(X \rightarrow Y)=\min _{U: U \Perp X} H(Y \mid U)-I(X ; Y)
$$

is closely related to one-shot channel simulation [36] and lossy source coding, and can be expressed as

$$
\begin{aligned}
\Psi(X \rightarrow Y) & =H(Y \mid X)-\max \left\{v_{Y}: v \in \mathscr{I}_{X Y}, v_{X}=0\right\} \\
& =\min \left\{R_{2}: R_{0}^{4} \in \mathscr{R}, R_{0}+R_{4}=H(Y \mid X)\right\}-I(X ; Y) \\
& =\min \left\{R_{2}: R_{0}^{4} \in \mathscr{R}, R_{4}=0, R_{0}=H(Y \mid X)\right\}-I(X ; Y) \\
& =-\psi_{\mathscr{I}_{X Y}}^{\prime}(-2,0,1 ; 0,1,-1) .
\end{aligned}
$$

In the EGW system, $\Psi(X \rightarrow Y)$ corresponds to the setting with only decoders 2,4 and $M_{4}=\emptyset$ (since it is better to allocate the rate to $R_{0}$ instead of $\left.R_{4}\right)$, and we restrict $R_{0}=H(Y \mid X)$. The value of $\Psi(X \rightarrow Y)+I(X ; Y)$ is the rate of the additional information $M_{2}$ that decoder 2 needs, in order to compensate the lack of side information compared to decoder 4.

Minimum communication rate for distributed channel synthesis with common randomness rate $t$ [13], [14]

$$
C(t, X \rightarrow Y)=\min _{U: X-U-Y} \max \{I(X ; U), I(X, Y ; U)-t\}
$$

can be expressed as

$$
\begin{aligned}
C(t, X \rightarrow Y) & =\min \left\{\max \left\{v_{X}, v_{X Y}-t\right\}: v \in \mathscr{I}_{X Y}, v_{X}+v_{Y}-v_{X Y}=I(X ; Y)\right\} \\
& =\min \left\{\max \left\{H(X)-R_{1}, R_{0}-t\right\}: R_{0}^{4} \in \mathscr{R}, R_{0}+R_{1}+R_{2}=H(X, Y)\right\}
\end{aligned}
$$




\section{A. New information theoretic quantities}

We now present three new quantities which arise as extreme points of $\mathscr{I}_{X Y}$. These extreme points concern the case in which decoders 3 and 4 are active in the EGW system. Note that they are all maximizations of the interaction information $I(X ; Y \mid U)-I(X ; Y)$ under various constraints. They can be considered as distances from extreme dependency, in the sense that they are equal to zero only under certain conditions of extreme dependency.

Maximal interaction information is defined as

$$
G_{\mathrm{NNI}}(X ; Y)=\max _{p_{U \mid X Y}} I(X ; Y \mid U)-I(X ; Y) .
$$

It can be shown that

$$
\begin{aligned}
G_{\mathrm{NNI}}(X ; Y) & =H(X \mid Y)+H(Y \mid X)-\min _{U: H(Y \mid X, U)=H(X \mid Y, U)=0} I(X, Y ; U) \\
& =\max \left\{v_{X Y}-v_{X}-v_{Y}: v \in \mathscr{I}_{X Y}\right\} \\
& =H(X \mid Y)+H(Y \mid X)-\min \left\{R_{0}+R_{3}+R_{4}: R_{0}^{4} \in \mathscr{R}\right\} \\
& =H(X \mid Y)+H(Y \mid X)-\min \left\{R_{0}: R_{0}^{4} \in \mathscr{R}, R_{3}=R_{4}=0\right\} \\
& =\psi_{\mathscr{I}_{X Y}}(-1,-1,1) .
\end{aligned}
$$

The maximal interaction information concerns the sum-rate of the EGW system with only decoders 3,4 . Note that it is always better to allocate the rates $R_{3}, R_{4}$ to $R_{0}$ instead, hence we can assume $R_{3}=R_{4}=0$ (which corresponds to $H(Y \mid X, U)=$ $H(X \mid Y, U)=0)$. The quantity $H(X \mid Y)+H(Y \mid X)-G_{\mathrm{NNI}}(X ; Y)$ is the maximum rate in the lossless causal version of the complementary delivery setup [7].

Asymmetric private interaction information is defined as

$$
G_{\mathrm{PNI}}(X \rightarrow Y)=\max _{U: U \Perp X} I(X ; Y \mid U)-I(X ; Y) .
$$

It can be shown that

$$
\begin{aligned}
G_{\mathrm{PNI}}(X \rightarrow Y) & =H(Y \mid X)-\min _{U: U \Perp X, H(Y \mid X, U)=0} I(Y ; U) \\
& =H(Y \mid X)-\min \left\{v_{Y}: v \in \mathscr{I}_{X Y}, v_{X}=0, v_{X Y}=H(Y \mid X)\right\} \\
& =H(X \mid Y)-\min \left\{R_{3}: R_{0}^{4} \in \mathscr{R}, R_{0}+R_{4}=H(Y \mid X)\right\} \\
& =H(X \mid Y)-\min \left\{R_{3}: R_{0}^{4} \in \mathscr{R}, R_{4}=0, R_{0}=H(Y \mid X)\right\} \\
& =\psi_{\mathscr{I}_{X Y}}^{\prime}(-1,0,0 ; 0,-1,1) .
\end{aligned}
$$

The asymmetric private interaction information is the opposite of excess functional information defined in [10] in which $I(Y ; U)$ is maximized instead. Another operational meaning of $G_{\mathrm{PNI}}$ is the generation of random variables with a privacy constraint. Suppose Alice observes $X$ and wants to generate $Y \sim p_{Y \mid X}(\cdot \mid X)$. However, she does not have any private randomness and can only access public randomness $W$, which is also available to Eve. Her goal is to generate $Y$ as a function of $X$ and $W$, while minimizing Eve's knowledge on $Y$ measured by $I(Y ; W)$. The minimum $I(Y ; W)$ is $H(Y \mid X)-G_{\mathrm{PNI}}(X \rightarrow$ $Y)$.

Symmetric private interaction information is defined as

$$
G_{\mathrm{PPI}}(X ; Y)=\max _{U: U \Perp X, U \Perp Y} I(X ; Y \mid U)-I(X ; Y) .
$$

It can be shown that

$$
\begin{aligned}
G_{\mathrm{PPI}}(X ; Y) & =\max _{U: U \Perp X, U \Perp Y} I(X, Y ; U) \\
& =\max \left\{v_{X Y}: v \in \mathscr{I}_{X Y}, v_{X}=v_{Y}=0\right\} \\
& =\max \left\{R_{0}: R_{0}^{4} \in \mathscr{R}, R_{0}+R_{3}=H(X \mid Y), R_{0}+R_{4}=H(Y \mid X)\right\} \\
& =\psi_{\mathscr{I}_{X Y}}^{\prime}(-1,-1,0 ; 0,0,1) .
\end{aligned}
$$

Intuitively, $G_{\mathrm{PPI}}$ captures the maximum amount of information one can disclose about $(X, Y)$, such that an eavesdropper who only has one of $X$ or $Y$ would know nothing about the disclosed information. Another operational meaning of $G_{\mathrm{PNI}}$ is the generation of random variables with a privacy constraint (similar to that for $G_{\mathrm{PNI}}$ ). Suppose Alice observes $X$ and wants to generate $Y \sim p_{Y \mid X}(\cdot \mid X)$. She has access to public randomness $W$, which is also available to Eve. She also has access to private randomness. Her goal is to generate $Y$ using $X, W$ and her private randomness such that Eve has no knowledge on $Y$ (i.e., $I(Y ; W)=0$ ), while minimizing the amount of private randomness used measured by $H(Y \mid X, W)$ (note that if Alice 


\begin{tabular}{|c|c|c|c|}
\hline $\begin{array}{l}\text { Active } \\
\text { decoders } \\
\text { in EGW }\end{array}$ & Information quantity & Objective and constraints in EGW & $\begin{array}{l}\text { Support fen. rep. } \\
\left(\psi=\psi_{\mathscr{I}_{X Y}}\right)\end{array}$ \\
\hline \multirow{5}{*}{1,2} & Wyner's CI [2] & $\min R_{0}: R_{0}+R_{1}+R_{2}=H(X, Y)$ & $-\psi^{\prime}(1,1,-1 ; 0,0,-1)$ \\
\hline & Gács-Körner CI [3], 25] & $\max R_{0}: R_{0}+R_{1}=H(X), R_{0}+R_{2}=H(Y)$ & $\psi^{\prime}(1,1,-2 ; 0,0,1)$ \\
\hline & Necessary conditional entropy [4, 27] & $\min R_{0}: R_{0}+R_{2}=H(Y), R_{1}=H(X \mid Y)$ & $-\psi^{\prime}(1,2,-2 ; 1,0,-1)$ \\
\hline & Info. bottleneck 5] & $\min R_{0}: R_{0}+R_{2}=H(Y), R_{1} \leq H(X)-t$ & none \\
\hline & Comm. rate for channel synthesis [13], [14] & $\min \max \left\{H(X)-R_{1}, R_{0}-t\right\}: R_{0}+R_{1}+R_{2}=H(X, Y)$ & none \\
\hline \multirow{4}{*}{$\begin{array}{c}1,3 \\
\text { or } 2,4\end{array}$} & Körner graph entropy [8] & $\min R_{0}: R_{0}+R_{1}=H(X), R_{3}=0$ & $-\psi^{\prime}(1,-1,0 ;-1,0,0)$ \\
\hline & Excess functional info. [10] & $\min R_{2}-I(X ; Y): R_{4}=0, R_{0}=H(Y \mid X)$ & $-\psi^{\prime}(-2,0,1 ; 0,1,-1)$ \\
\hline & Max. rate for perfect privacy [9], 29] & $\max R_{0}: R_{0}+R_{2}=H(Y), R_{0}+R_{4}=H(Y \mid X)$ & $\psi^{\prime}(-1,1,-1 ; 0,1,0)$ \\
\hline & Privacy funnel [9] & $\min R_{0}+R_{4}-H(Y \mid X): R_{0}+R_{2}=H(Y), R_{0} \geq t$ & none \\
\hline \multirow{3}{*}{3,4} & Maximal interaction info. & $\max H(X \mid Y)+H(Y \mid X)-R_{0}: R_{3}=R_{4}=0$ & $\psi(-1,-1,1)$ \\
\hline & Asymm. private interaction info. & $\max H(X \mid Y)-R_{3}: R_{4}=0, R_{0}=H(Y \mid X)$ & $\psi^{\prime}(-1,0,0 ; 0,-1,1)$ \\
\hline & Symm. private interaction info. & $\max R_{0}: R_{0}+R_{3}=H(X \mid Y), R_{0}+R_{4}=H(Y \mid X)$ & $\psi^{\prime}(-1,-1,0 ; 0,0,1)$ \\
\hline
\end{tabular}

Table I

EXTREME POINTS OF $\mathscr{I}_{X Y}$ AND THE CORRESPONDING EXTREME POINTS IN THE EGW, AND THEIR SUPPORT FUNCTION REPRESENTATIONS.

can flip fair coins for the private randomness, then by Knuth-Yao algorithm [37 the expected number of flips is bounded by $H(Y \mid X, W)+2)$. The minimum $H(Y \mid X, W)$ is $H(Y \mid X)-G_{\mathrm{PPI}}(X ; Y)$.

We now list several properties of $G_{\mathrm{NNI}}, G_{\mathrm{PNI}}$ and $G_{\mathrm{PPI}}$.

Proposition 3. $G_{\mathrm{NNI}}, G_{\mathrm{PNI}}$ and $G_{\mathrm{PPI}}$ satisfies

1) Bounds.

$$
0 \leq G_{\mathrm{PPI}}(X ; Y) \leq G_{\mathrm{PNI}}(X \rightarrow Y) \leq G_{\mathrm{NNI}}(X ; Y) \leq \min \{H(X \mid Y), H(Y \mid X)\}
$$

2) Conditions for zero.

- $G_{\mathrm{NNI}}(X ; Y)=0$ if and only if the characteristic bipartite graph of $X, Y$ (i.e. vertices $\mathcal{X} \cup \mathcal{Y}$ with edge $(x, y)$ if $p(x, y)>0)$ does not contain paths of length 3 , or equivalently, $p(x \mid y)=1$ or $p(y \mid x)=1$ for all $x, y$ such that $p(x, y)>0$.

- $G_{\mathrm{PNI}}(X \rightarrow Y)=0$ if and only if $G_{\mathrm{NNI}}(X ; Y)=0$.

- $G_{\mathrm{PPI}}(X ; Y)=0$ if and only if the characteristic bipartite graph of $X, Y$ does not contain cycles.

3) Condition for maximum. If $H(X)=H(Y)$, then the following statements are equivalent:

- $G_{\mathrm{NNI}}(X ; Y)=H(Y \mid X)$.

- $G_{\mathrm{PNI}}(X \rightarrow Y)=H(Y \mid X)$.

- $G_{\mathrm{PPI}}(X ; Y)=H(Y \mid X)$.

- $p(x)=p(y)$ for all $x, y$ such that $p(x, y)>0$.

4) Lower bound for independent $X, Y$. If $X \Perp Y$,

$$
G_{\mathrm{PPI}}(X ; Y) \geq \mathrm{E}[-\log \max \{p(X), p(Y)\}]-1 .
$$

5) Superadditivity. If $\left(X_{1}, Y_{1}\right)$ is independent of $\left(X_{2}, Y_{2}\right)$, then

$$
G_{\mathrm{NNI}}\left(X_{1}, X_{2} ; Y_{1}, Y_{2}\right) \geq G_{\mathrm{NNI}}\left(X_{1} ; Y_{1}\right)+G_{\mathrm{NNI}}\left(X_{2} ; Y_{2}\right) \text {. }
$$

Similar for $G_{\mathrm{PNI}}$ and $G_{\mathrm{PPI}}$.

The proof of this proposition is given in Appendix C. 


\section{EXTEnded GRAY-Wyner System With NonCAUSAl COMPLEMENTARy Side Information}

In this section we establish the rate region $\mathscr{R}^{\prime}$ for the EGW system with complementary noncausal side information at decoders 3 and 4 (noncausal EGW), that is, decoder 3 recovers $X^{n}$ from $\left(M_{0}, M_{3}, Y^{n}\right)$ and decoder 4 similarly recovers $Y^{n}$ from $\left(M_{0}, M_{4}, X^{n}\right)$. We show that $\mathscr{R}^{\prime}$ can be expressed in terms of the Gray-Wyner region $\mathscr{R}_{\mathrm{GW}}$, hence it contains fewer interesting extreme points compared to $\mathscr{R}$. This is the reason we emphasized the causal side information in this paper. We further show that $\mathscr{R}^{\prime}$ is related to the asymptotic mutual information region defined as

$$
\mathscr{I}_{X Y}^{\infty}=\bigcup_{n=1}^{\infty} \frac{1}{n} \mathscr{I}_{X^{n}, Y^{n}}
$$

where $\left(X^{n}, Y^{n}\right)$ is i.i.d. with $\left(X_{1}, Y_{1}\right) \sim p_{X Y}$. Note that $\mathscr{I}_{X Y}^{\infty}$ may not be closed (unlike $\mathscr{I}_{X Y}$ which is always closed).

The following gives the rate region for the noncausal EGW.

Theorem 2. The optimal rate region $\mathscr{R}^{\prime}$ for the extended Gray-Wyner system with noncausal complementary side information is the set of rate tuples $\left(R_{0}, R_{1}, R_{2}, R_{3}, R_{4}\right)$ such that

$$
\begin{aligned}
& R_{0} \geq I(X, Y ; U), \\
& R_{1} \geq H(X \mid U), \\
& R_{2} \geq H(Y \mid U), \\
& R_{3} \geq H(X \mid U)-H(Y), \\
& R_{4} \geq H(Y \mid U)-H(X), \\
& R_{0}+ R_{3} \geq H(X \mid Y), \\
& R_{0}+R_{4} \geq H(Y \mid X), \\
& R_{2}+R_{3} \geq H(X \mid U), \\
& R_{1}+R_{4} \geq H(Y \mid U), \\
& R_{0}+R_{2}+R_{3} \geq H(X, Y), \\
& R_{0}+R_{1}+R_{4} \geq H(X, Y)
\end{aligned}
$$

for some $p_{U \mid X Y}$, where $|\mathcal{U}| \leq|\mathcal{X}| \cdot|\mathcal{Y}|+2$.

The proof is given in Appendix D] Then we characterize the closure of $\mathscr{I}_{X Y}^{\infty}$. We show that $\operatorname{cl}\left(\mathscr{I}_{X Y}^{\infty}\right), \mathscr{R}^{\prime}$ and the the Gray-Wyner region $\mathscr{R}_{\mathrm{GW}}$ can be expressed in terms of each other.

Proposition 4. The closure of $\mathscr{I}_{X Y}^{\infty}$, the rate region $\mathscr{R}^{\prime}$ for the noncausal EGW and the Gray-Wyner region $\mathscr{R}_{\mathrm{GW}}$ satisfy:

1) Characterization of $\operatorname{cl}\left(\mathscr{I}_{X Y}^{\infty}\right)$.

$$
\begin{aligned}
\operatorname{cl}\left(\mathscr{I}_{X Y}^{\infty}\right) & =\left(\mathscr{I}_{X Y}+(-\infty, 0] \times(-\infty, 0] \times[0, \infty)\right) \cap \mathscr{I}_{X Y}^{\mathrm{o}} \\
& =\left(\mathscr{I}_{X Y}+\{(t, t, t): t \leq 0\}\right) \cap([0, \infty) \times[0, \infty) \times \mathbb{R}) .
\end{aligned}
$$

2) Equivalence between $\operatorname{cl}\left(\mathscr{I}_{X Y}^{\infty}\right)$ and $\mathscr{R}^{\prime}$.

$$
\mathscr{R}^{\prime}=\bigcup_{v \in \operatorname{cl}\left(\mathscr{I}_{X Y}^{\infty}\right)}\left\{\left(v_{X Y}, H(X)-v_{X}, H(Y)-v_{Y}, H(X \mid Y)-v_{X Y}+v_{Y}, H(Y \mid X)-v_{X Y}+v_{X}\right)\right\}+[0, \infty)^{5},
$$

and

$$
\operatorname{cl}\left(\mathscr{I}_{X Y}^{\infty}\right)=\left\{v \in \mathbb{R}^{3}:\left(v_{X Y}, H(X)-v_{X}, H(Y)-v_{Y}, H(X \mid Y)-v_{X Y}+v_{Y}, H(Y \mid X)-v_{X Y}+v_{X}\right) \in \mathscr{R}^{\prime}\right\} .
$$

3) Equivalence between $\operatorname{cl}\left(\mathscr{I}_{X Y}^{\infty}\right)$ and $\mathscr{R}_{\mathrm{GW}}$.

$$
\mathscr{R}_{\mathrm{GW}}=\bigcup_{v \in \operatorname{cl}\left(\mathscr{I}_{X Y}^{\infty}\right)}\left\{\left(v_{X Y}, H(X)-v_{X}, H(Y)-v_{Y}\right)\right\}+[0, \infty)^{3},
$$

and

$$
\operatorname{cl}\left(\mathscr{I}_{X Y}^{\infty}\right)=\left\{v \in \mathscr{I}_{X Y}^{\mathrm{o}}:\left(v_{X Y}, H(X)-v_{X}, H(Y)-v_{Y}\right) \in \mathscr{R}_{\mathrm{GW}}\right\}
$$

The proof is given in Appendix E] Note that Proposition 4 does not characterize $\mathscr{I}_{X Y}^{\infty}$ completely since it does not specify which boundary points are in $\mathscr{I}_{X Y}^{\infty}$. 


\section{APPENDIX}

A. Proof of the converse of Theorem 1

To prove the converse, let $U_{i}=\left(M_{0}, X^{i-1}, Y^{i-1}\right)$. Consider

$$
\begin{aligned}
n R_{0} & \geq I\left(X^{n}, Y^{n} ; M_{0}\right) \\
& =\sum_{i=1}^{n} I\left(X_{i}, Y_{i} ; M_{0} \mid X^{i-1}, Y^{i-1}\right) \\
& =\sum_{i=1}^{n} I\left(X_{i}, Y_{i} ; M_{0}, X^{i-1}, Y^{i-1}\right) \\
& =\sum_{i=1}^{n} I\left(X_{i}, Y_{i} ; U_{i}\right), \\
n R_{1} & \geq H\left(M_{1} \mid M_{0}\right) \\
& \geq I\left(X^{n} ; M_{1} \mid M_{0}\right) \\
& =H\left(X^{n} \mid M_{0}\right)-H\left(X^{n} \mid M_{0}, M_{1}\right) \\
& =\sum_{i=1}^{n} H\left(X_{i} \mid M_{0}, X^{i-1}\right)-H\left(X^{n} \mid M_{0}, M_{1}\right) \\
& \geq \sum_{i=1}^{n} H\left(X_{i} \mid M_{0}, X^{i-1}, Y^{i-1}\right)-H\left(X^{n} \mid M_{0}, M_{1}\right) \\
& \geq \sum_{i=1}^{n} H\left(X_{i} \mid M_{0}, X^{i-1}, Y^{i-1}\right)-\log |\mathcal{X}| \sum_{i=1}^{n} \mathrm{P}\left\{X_{i} \neq \hat{X}_{1, i}\right\}-1 \\
& \geq \sum_{i=1}^{n} H\left(X_{i} \mid U_{i}\right)-o(n),
\end{aligned}
$$

where the last inequality follows by Fano's inequality. Similarly $n R_{2} \geq \sum_{i} H\left(Y_{i} \mid U_{i}\right)-o(n)$. Next, consider

$$
\begin{aligned}
n R_{3} & \geq H\left(M_{3} \mid M_{0}\right) \\
& \geq I\left(X^{n}, Y^{n} ; M_{3} \mid M_{0}\right) \\
& =\sum_{i=1}^{n} I\left(X_{i}, Y_{i} ; M_{3} \mid M_{0}, X^{i-1}, Y^{i-1}\right) \\
& \geq \sum_{i=1}^{n} I\left(X_{i} ; M_{3} \mid M_{0}, X^{i-1}, Y^{i}\right) \\
& =\sum_{i=1}^{n}\left(H\left(X_{i} \mid M_{0}, X^{i-1}, Y^{i}\right)-H\left(X_{i} \mid M_{0}, M_{3}, X^{i-1}, Y^{i}\right)\right) \\
& =\sum_{i=1}^{n} H\left(X_{i} \mid Y_{i}, U_{i}\right)-\sum_{i=1}^{n} H\left(X_{i} \mid M_{0}, M_{3}, X^{i-1}, Y^{i}\right) \\
& \geq \sum_{i=1}^{n} H\left(X_{i} \mid Y_{i}, U_{i}\right)-\log |\mathcal{X}| \sum_{i=1}^{n} \mathrm{P}\left\{X_{i} \neq \hat{X}_{3, i}\right\}-1 \\
& =\sum_{i=1}^{n} H\left(X_{i} \mid Y_{i}, U_{i}\right)-o(n),
\end{aligned}
$$

where the last inequality follows by Fano's inequality since $\hat{X}_{3, i}$ is a function of $M_{0}, M_{3}, Y^{i}$. Similarly $n R_{4} \geq \sum_{i} H\left(Y_{i} \mid X_{i}, U_{i}\right)-$ $o(n)$. Hence the point $\left(R_{0}+\epsilon, \ldots, R_{4}+\epsilon\right)$ is in the convex hull of $\mathscr{R}$ for any $\epsilon>0$. From (2), $\mathscr{R}$ is the increasing hull of an affine transformation of $\mathscr{I}_{X Y}$, and thus is convex.

To prove the cardinality bound, we apply Fenchel-Eggleston-Carathéodory theorem [38], [39] on the $(|\mathcal{X}||\mathcal{Y}|+2)$-dimensional vectors with entries $H(X \mid U=u), H(Y \mid U=u), H(X, Y \mid U=u)$ and $p(x, y \mid u)$ for $u \in\{1, \ldots,|\mathcal{U}|\},(x, y) \in\{1, \ldots,|\mathcal{X}|\} \times$ $\{1, \ldots,|\mathcal{Y}|\} \backslash(|\mathcal{X}|,|\mathcal{Y}|)$; see [16], [19]. 


\section{B. Proof of Proposition 2}

1) To see that $\mathscr{I}_{X Y}$ is convex, for any $U_{0}, U_{1}$ and $\lambda \in[0,1]$, let $Q \sim \operatorname{Bern}(\lambda)$ be independent of $X, Y, U_{0}, U_{1}$, and let $U=\left(Q, U_{Q}\right)$. Then $I(X ; U)=(1-\lambda) I\left(X ; U_{0}\right)+\lambda I\left(X ; U_{1}\right)$ (similarly for the other two quantities). Compactness will be proved later.

2) The outer bound follows directly from the properties of entropy and mutual information.

3) For the inner bound, the first 4 points can be obtained by substituting $U=\emptyset, X, Y,(X, Y)$ respectively. For the last point, by the functional representation lemma [40, p. 626], let $V \Perp X$ such that $H(Y \mid X, V)=0$. Again by the functional representation lemma, let $W \Perp(Y, V)$ such that $H(X \mid Y, V, W)=0$. Let $U=(V, W)$, then $I(X, Y ; U)-I(X ; U)=$ $H(Y \mid X)-H(Y \mid X, U)=H(Y \mid X), I(X, Y ; U)-I(Y ; U)=H(X \mid Y)-H(X \mid Y, U)=H(X \mid Y)$, and

$$
\begin{aligned}
I(X, Y ; U) & =I(X, Y ; V, W) \\
& =I(X, Y ; V)+I(X, Y ; W \mid V) \\
& =I(Y ; V \mid X)+I(X ; W \mid Y, V) \\
& \leq H(Y \mid X)+H(X \mid Y) .
\end{aligned}
$$

Hence there exists $t \leq H(Y \mid X)+H(X \mid Y)$ such that $(t-H(Y \mid X), t-H(X \mid Y), t) \in \mathscr{I}_{X Y}$ (by substituting $t=$ $I(X, Y ; U)$ ). Taking convex combination of this point and $(H(X), H(Y), H(X, Y)) \in \mathscr{I}_{X Y}$, we have $(H(X \mid Y), H(Y \mid X), H(X \mid Y)-$ $H(Y \mid X)) \in \mathscr{I}_{X Y}$.

The existence of $0 \leq \epsilon_{1} \leq \log I(X ; Y)+4$ such that $\left(0, H(Y \mid X)-\epsilon_{1}, H(Y \mid X)\right) \in \mathscr{I}_{X Y}$ can be proved by substituting $\epsilon_{1}=\Psi(X \rightarrow Y)$ and invoking the strong functional representation lemma [10].

4) The superadditivity property can be obtained from considering $U=\left(U_{1}, U_{2}\right)$, where $\left(I\left(X_{i} ; U_{i}\right), I\left(Y_{i} ; U_{i}\right), I\left(X_{i}, Y_{i} ; U_{i}\right)\right) \in$ $\mathscr{I}_{X_{i}, Y_{i}}$.

5) The data processing property can be obtained from considering $U$ where $\left(I\left(X_{1} ; U\right), I\left(Y_{1} ; U\right), I\left(X_{1}, Y_{1} ; U\right)\right) \in \mathscr{I}_{X_{1}, Y_{1}}$.

6) The cardinality bound can be proved using Fenchel-Eggleston-Carathéodory theorem using the same arguments as in the converse proof of Theorem 1 Compactness follows from the fact that mutual information is a continuous function, and the set of conditional pmfs $p_{U \mid X Y}$ with $|\mathcal{U}| \leq|\mathcal{X}| \cdot|\mathcal{Y}|+2$ is a compact set.

7) The relation to Gray-Wyner region and region of tension follows from the definitions of the regions.

\section{Proof of Proposition 3}

1) To prove the bound, note that $I(X ; Y \mid U) \leq H(X)$, hence $I(X ; Y \mid U)-I(X ; Y) \leq H(X \mid Y)$, $G_{\mathrm{NNI}} \leq H(X \mid Y)$.

2) We first prove that if there does not exist length 3 paths in the bipartite graph, then $G_{\mathrm{NNI}}(X ; Y)=G_{\mathrm{PNI}}(X ; Y)=0$. Let $Q$ achieves the Gács-Körner common information, i.e., $Q$ represents which connected component the edge $(X, Y)$ lies in. If the bipartite graph does not contain length 3 paths, every connected component is a star, i.e., for each $q$, either $H(X \mid Q=q)=0$ or $H(Y \mid Q=q)=0$. Then $I(X ; Y)=H(Q)+I(X ; Y \mid Q)=H(Q)$, and $I(X ; Y \mid U)=$ $H(Q \mid U)+I(X ; Y \mid Q, U)=H(Q \mid U) \leq H(Q)$ for any $U$. Hence $G_{\mathrm{NNI}}(X ; Y)=G_{\mathrm{PNI}}(X ; Y)=0$.

We then prove that if there exist a length 3 path in the bipartite graph, then $G_{\mathrm{NNI}}(X ; Y) \geq G_{\mathrm{PNI}}(X ; Y)>0$. Assume $p\left(x_{1}, y_{1}\right), p\left(x_{1}, y_{2}\right), p\left(x_{2}, y_{1}\right)>0$. Let $U \in\{1,2\}$,

$$
p(u \mid x, y)= \begin{cases}1 / 2+\epsilon / p\left(x_{1}, y_{1}\right) & \text { if }(x, y, u)=\left(x_{1}, y_{1}, 1\right) \\ 1 / 2-\epsilon / p\left(x_{1}, y_{1}\right) & \text { if }(x, y, u)=\left(x_{1}, y_{1}, 2\right) \\ 1 / 2-\epsilon / p\left(x_{1}, y_{2}\right) & \text { if }(x, y, u)=\left(x_{1}, y_{2}, 1\right) \\ 1 / 2+\epsilon / p\left(x_{1}, y_{2}\right) & \text { if }(x, y, u)=\left(x_{1}, y_{2}, 2\right) \\ 1 / 2 & \text { otherwise, }\end{cases}
$$

where $\epsilon>0$ is small enough such that the above is a valid conditional pmf. One can verify that $U \Perp X$. Since $p_{U \mid X Y}\left(1 \mid x_{1}, y_{1}\right)=1 / 2+\epsilon / p\left(x_{1}, y_{1}\right) \neq 1 / 2=p_{U \mid X Y}\left(1 \mid x_{2}, y_{1}\right), X$ and $U$ are not conditionally independent given $Y$. Hence $I(X ; Y \mid U)-I(X ; Y)=I(X ; U \mid Y)>0$.

We then prove that if $G_{\mathrm{PPI}}(X ; Y)>0$, then there exists a cycle in the bipartite graph. Let $U$ satisfies $U \Perp X, U \Perp Y$ and $I(X ; Y \mid U)>I(X ; Y)$. Since $U$ is not independent of $X, Y$, there exists $x_{1}, y_{1}, u$ such that $p\left(x_{1}, y_{1} \mid u\right)>p\left(x_{1}, y_{1}\right)$. Since $\sum_{y^{\prime}} p\left(x_{1}, y^{\prime} \mid u\right)=p\left(x_{1} \mid u\right)=p\left(x_{1}\right)=\sum_{y^{\prime}} p\left(x_{1}, y^{\prime}\right)$, there exists $y_{2} \neq y_{1}$ such that $p\left(x_{1}, y_{2} \mid u\right)<p\left(x_{1}, y_{2}\right)$. Since $\sum_{x^{\prime}} p\left(x^{\prime}, y_{2} \mid u\right)=p\left(y_{2} \mid u\right)=p\left(y_{2}\right)=\sum_{x^{\prime}} p\left(x^{\prime}, y_{2}\right)$, there exists $x_{2} \neq x_{1}$ such that $p\left(x_{2}, y_{2} \mid u\right)>p\left(x_{2}, y_{2}\right)$. Continue this process until we return to a visited $x, y$ pair, i.e., $\left(x_{a}, y_{a}\right)=\left(x_{b}, y_{b}\right)$ for $a<b$. Then $y_{a}, x_{a}, y_{a+1}, x_{a+1}, \ldots, x_{b-1}, y_{b}$ forms a cycle.

We then prove that if there exist a cycle in the bipartite graph, then $G_{\mathrm{PPI}}(X ; Y)>0$. Let $y_{1}, x_{1}, y_{2}, x_{2}, \ldots, x_{a}, y_{a+1}=y_{1}$ 
be a cycle. Let $U \in\{1,2\}$,

$$
p(u \mid x, y)= \begin{cases}1 / 2+\epsilon / p\left(x_{i}, y_{i}\right) & \text { if }(x, y, u)=\left(x_{i}, y_{i}, 1\right) \\ 1 / 2-\epsilon / p\left(x_{i}, y_{i}\right) & \text { if }(x, y, u)=\left(x_{i}, y_{i}, 2\right) \\ 1 / 2-\epsilon / p\left(x_{i}, y_{i+1}\right) & \text { if }(x, y, u)=\left(x_{i}, y_{i+1}, 1\right) \\ 1 / 2+\epsilon / p\left(x_{i}, y_{i+1}\right) & \text { if }(x, y, u)=\left(x_{i}, y_{i+1}, 2\right) \\ 1 / 2 & \text { otherwise, }\end{cases}
$$

where $\epsilon>0$ is small enough such that the above is a valid conditional pmf. One can verify that $U \Perp X$ and $U \Perp Y$. Since $p_{U \mid X Y}\left(1 \mid x_{1}, y_{1}\right)>1 / 2>p_{U \mid X Y}\left(1 \mid x_{1}, y_{2}\right), U$ is not independent of $X, Y$. Hence $I(X ; Y \mid U)-I(X ; Y)=$ $I(X, Y ; U)>0$.

3) We then prove that if $H(X)=H(Y)$ and $p(x)=p(y)$ for all $x, y$ such that $p(x, y)>0$, then $G_{\mathrm{PPI}}(X ; Y)=$ $H(Y \mid X)$. Let $Q$ achieves the Gács-Körner common information, and let $\mathcal{X}_{q}=\{x: p(x \mid q)>0\}, \mathcal{Y}_{q}=\{y: p(y \mid q)>0\}$, then $X\left|\{Q=q\} \sim \operatorname{Unif}\left(\mathcal{X}_{q}\right), Y\right|\{Q=q\} \sim \operatorname{Unif}\left(\mathcal{Y}_{q}\right)$ and $\left|\mathcal{X}_{q}\right|=\left|\mathcal{Y}_{q}\right|$ for all $q$. Applying Birkhoff-von Neumann theorem on the submatrix of $p(x, y)$ with rows $\mathcal{X}_{q}$ and columns $\mathcal{Y}_{q}$, there exists $U_{q}$ such that $p(x, y \mid q)=$ $\sum_{u} p_{U_{q}}(u) p_{X Y \mid U_{q} Q}(x, y \mid u, q), p_{X \mid U_{q} Q}(x \mid u, q)=p_{Y \mid U_{q} Q}(y \mid u, q)=1 /\left|\mathcal{X}_{q}\right|$ and $p_{X Y \mid U_{q} Q}(x, y \mid u, q) \in\left\{0,1 /\left|\mathcal{X}_{q}\right|\right\}$ for all $x, y, u$. Let $U=\left\{U_{q}\right\}_{q \in \mathcal{Q}}$, where $U_{q}$ are assumed to be independent across $q$. Then for any $x$ and $u=\left\{u_{q}\right\}$,

$$
\begin{aligned}
p\left(x \mid\left\{u_{q}\right\}\right) & =p\left(x, q \mid\left\{u_{q}\right\}\right) \\
& =p(q) p\left(x \mid u_{q}, q\right) \\
& =p(q) /\left|\mathcal{X}_{q}\right| \\
& =p(x),
\end{aligned}
$$

where $q=q(x)$ since $H(Q \mid X)=0$. Hence $U \Perp X$. Similarly $U \Perp Y$. Also since there is only one non-zero in $p_{X Y \mid U_{q} Q}(x, y \mid u, q)$ for different $x$, we have $H(X \mid Y, U)=0$. Similarly $H(Y \mid X, U)=0$. Hence $I(X ; Y \mid U)-I(X ; Y)=$ $I(Y ; U \mid X)-I(Y ; U)=H(Y \mid X)$.

We then prove that if $H(X)=H(Y)$ and $G_{\mathrm{NNI}}(X ; Y)=H(Y \mid X)$, then $p(x)=p(y)$ for all $x, y$ such that $p(x, y)>0$. Let $U$ satisfies $I(X ; Y \mid U)=I(X ; Y)+H(Y \mid X)=H(Y)$, then one can check that $U \Perp X, U \Perp Y, H(X \mid Y, U)=0$ and $H(Y \mid X, U)=0$. For any $x, y$ such that $p(x, y)>0$, let $u$ such that $p(x, y, u)>0$, then

$$
\begin{aligned}
p(x) & =p(x \mid u) \\
& =p(x \mid u) p(y \mid x, u) \\
& =p(y \mid u) p(x \mid y, u) \\
& =p(y) .
\end{aligned}
$$

4) We then prove the lower bound when $X, Y$ independent. Assume $X \Perp Y$. Assume $\mathcal{X}=\{1, \ldots,|\mathcal{X}|\}, Y=\{1, \ldots,|\mathcal{Y}|\}$, $X=F_{X}^{-1}(V), Y=F_{Y}^{-1}(W), V, W \sim$ Unif [0,1] independent. Let $U=V+W \bmod 1$, then $U \Perp X, U \Perp Y$.

$$
\begin{aligned}
H(Y \mid U, X) & =\sum_{x} p(x) \int_{0}^{1} H(Y \mid U=u, X=x) d u \\
& =\sum_{x} p(x) \int_{0}^{1} H\left(Y \mid W \in\left(\left[u-F_{X}(x), u-F_{X}(x-1)\right) \bmod 1\right)\right) d u \\
& =\sum_{x} p(x) \int_{0}^{1} H(Y \mid W \in([u, u+p(x)] \bmod 1)) d u \\
& =\sum_{x} p(x) \int_{0}^{1} \sum_{y} l(\mathrm{P}\{Y=y \mid W \in([u, u+p(x)] \bmod 1)\}) d u \\
& =\sum_{x} p(x) \int_{0}^{1} \sum_{y} l\left(p(x)^{-1}\left|\left[F_{Y}(y-1), F_{Y}(y)\right] \cap([u, u+p(x)] \bmod 1)\right|\right) d u \\
& =\sum_{x} p(x) \sum_{y} \int_{0}^{1} l\left(p(x)^{-1}|[0, p(y)] \cap([u, u+p(x)] \bmod 1)|\right) d u \\
& =-H(X)+\sum_{x, y} \int_{0}^{1} l(|[0, p(y)] \cap([u, u+p(x)] \bmod 1)|) d u
\end{aligned}
$$


where we write $A \bmod 1=\{a \bmod 1: a \in A\}$ and $|A|$ for the Lebesgue measure for $A \subseteq \mathbb{R}, l(t)=-t \log t$. Consider

$$
f(a, b)=\int_{0}^{1} l(|[0, b] \cap([u, u+a] \bmod 1)|) d u .
$$

If $b \leq a \leq 1$ and $a+b \leq 1$,

$$
\begin{aligned}
f(a, b) & =(a-b) l(b)+2 \int_{0}^{b} l(u) d u \\
& \leq(a-b) l(b)+2 b l(b / 2) \\
& =a l(b)+b^{2} \\
& =a b \log \frac{1}{b}+b^{2} \\
& \leq a b \log \frac{1}{b}+a b .
\end{aligned}
$$

If $b \leq a$ and $a+b>1$,

$$
\begin{aligned}
f(a, b) & =(a-b) l(b)+(a+b-1) l(a+b-1)+2 \int_{b+a-1}^{b} l(u) d u \\
& \leq(a-b) l(b)+(a+b-1) l(a+b-1)+2(1-a) l\left(b-\frac{1-a}{2}\right) \\
& \leq(a-b) l(b)+(1+b-a) l\left(\frac{b^{2}}{1+b-a}\right) \\
& =(a-b) l(b)+b^{2} \log \frac{1+b-a}{b^{2}} \\
& \leq(a-b) l(b)+b^{2} \log \frac{2 b}{b^{2}} \\
& =a l(b)+b^{2} \\
& \leq a b \log \frac{1}{b}+a b .
\end{aligned}
$$

Hence

$$
\begin{aligned}
I(X, Y ; U) & =H(X, Y)-H(Y \mid U, X)-H(X \mid U) \\
& =H(X, Y)-\sum_{x, y} f(p(x), p(y)) \\
& \geq H(X, Y)-\sum_{x, y}\left(p(x) p(y) \log \frac{1}{\min \{p(x), p(y)\}}+p(x) p(y)\right) \\
& =\mathrm{E}\left[\log \frac{1}{\max \{p(X), p(Y)\}}\right]-1 .
\end{aligned}
$$

5) The superadditivity property follows from the superadditivity of mutual information region.

\section{Proof of Theorem 2}

We first prove the achievability. Without loss of generality assume $H(X) \geq H(Y)$. Fix any point $v=\left(v_{X}, v_{Y}, v_{X Y}\right) \in \mathscr{I}_{X Y}$. Consider the region

$$
\mathscr{I}(v)=\left(\left(-\infty, v_{X}\right] \times\left(-\infty, v_{Y}\right] \times\left[v_{X Y}, \infty\right)\right) \cap \mathscr{I}_{X Y}^{\mathrm{o}} .
$$


It can be seen from Figure 2 that $\mathscr{I}(v)$ is a subset of the convex hull of the following 9 points:

$$
\begin{aligned}
& v, \\
& p_{1}=(0,0,0), \\
& p_{2}=(H(X), I(X ; Y), H(X)), \\
& p_{3}=(I(X ; Y), H(Y), H(Y)), \\
& p_{4}=(H(X), H(Y), H(X, Y)), \\
& p_{5}=(H(X \mid Y), 0, H(X \mid Y)), \\
& p_{6}=(0, H(Y \mid X), H(Y \mid X)), \\
& p_{7}=(0,0, H(Y \mid X)), \\
& p_{8}=(H(X)-H(Y), 0, H(X \mid Y)),
\end{aligned}
$$

i.e., $v$ together with the corner points of $\mathscr{I}_{X Y}^{\mathrm{o}}$ except $(I(X ; Y), I(X ; Y), I(X ; Y))$. We will prove that for any $w=$ $\left(w_{X}, w_{Y}, w_{X Y}\right) \in \mathscr{I}(v)$, the rate tuple $R(w)=\left(R_{0}(w), \ldots, R_{4}(w)\right)$,

$$
\begin{aligned}
& R_{0}(w)=w_{X Y}+\epsilon \\
& R_{1}(w)=H(X)-w_{X}+\epsilon \\
& R_{2}(w)=H(Y)-w_{Y}+\epsilon \\
& R_{3}(w)=H(X \mid Y)-w_{X Y}+w_{Y}+\epsilon, \\
& R_{4}(w)=H(Y \mid X)-w_{X Y}+w_{X}+\epsilon
\end{aligned}
$$

is achievable in the extended Gray-Wyner system with noncausal complementary side information for $\epsilon>0$. It suffices to prove the corner points $R(v), R\left(p_{1}\right), \ldots, R\left(p_{8}\right)$ are achievable.

$R(v)$ is achievable using the causal scheme in Theorem 11 To achieve $R\left(p_{1}\right), R\left(p_{2}\right), R\left(p_{3}\right)$ and $R\left(p_{4}\right)$, apply the causal scheme in Theorem 1 on $U \leftarrow \emptyset, U \leftarrow X, U \leftarrow Y$ and $U \leftarrow(X, Y)$, respectively.

To achieve $R\left(p_{5}\right)$, applying the strong functional representation lemma [10], there exists $V_{n} \Perp Y^{n}$ such that $H\left(X^{n} \mid Y^{n}, V_{n}\right)=$ 0 and $I\left(V_{n} ; Y^{n} \mid X^{n}\right) \leq \epsilon n / 2$ for $n$ large enough. We then apply the causal scheme on $X \leftarrow X^{n}, Y \leftarrow Y^{n}$ and $U \leftarrow V_{n}$. Similar for $R\left(p_{6}\right)$.

We now prove the achievability of $R\left(p_{7}\right)$. To generate the codebook, randomly partition $\mathcal{T}_{\epsilon^{\prime}}^{(n)}(X, Y)$ into bins $\mathcal{B}_{0}\left(m_{0}\right)$ of size $2^{n\left(H(X, Y)+\epsilon / 2-R_{0}\right)}$ for $m_{0} \in\left[1: 2^{n R_{0}}\right]$. Further randomly partition the bin $\mathcal{B}_{0}\left(m_{0}\right)$ into $\mathcal{B}_{3}\left(m_{0}, m_{3}\right)$ of size $2^{n\left(H(X, Y)+\epsilon / 2-R_{0}-R_{3}\right)}$ for $m_{3} \in\left[1: 2^{n R_{3}}\right]$.

To encode $x^{n}, y^{n}$, find $m_{0}, m_{3}$ such that $\left(x^{n}, y^{n}\right) \in \mathcal{B}_{3}\left(m_{0}, m_{3}\right)$. Directly encode $x^{n}, y^{n}$ into $m_{1}$ and $m_{2}$ respectively.

Decoder 3 receives $m_{0}, m_{3}, y^{n}$ and output the unique $\hat{x}^{n}$ such that $\left(\hat{x}^{n}, y^{n}\right) \in \mathcal{B}_{3}\left(m_{0}, m_{3}\right)$. The probability of error vanishes if $H(Y)>H(X, Y)+\epsilon / 2-R_{0}-R_{3}$, which is guaranteed by the definition of $R\left(p_{7}\right)$. Decoder 4 receives $m_{0}, x^{n}$ and output the unique $\hat{y}^{n}$ such that $\left(x^{n}, \hat{y}^{n}\right) \in \mathcal{B}_{0}\left(m_{0}\right)$. The probability of error vanishes if $H(X)>H(X, Y)+\epsilon / 2-R_{0}$, which is guaranteed by the definition of $R\left(p_{7}\right)$.

The achievability of $R\left(p_{8}\right)$ is similar to that of $R\left(p_{7}\right)$. To generate the codebook, randomly partition $\mathcal{T}_{\epsilon^{\prime}}^{(n)}(X, Y)$ into bins $\mathcal{B}_{0}\left(m_{0}\right)$ of size $2^{n\left(H(X, Y)+\epsilon / 2-R_{0}\right)}$ for $m_{0} \in\left[1: 2^{n R_{0}}\right]$. Given $m_{0}$, assign indices $m_{1}$ to the sequences in the bin $\mathcal{B}_{0}\left(m_{0}\right)$ for $m_{1} \in\left[1: 2^{n R_{1}}\right]$. This is possible if $R_{1} \geq H(X, Y)+\epsilon / 2-R_{0}$, which is guaranteed by the definition of $R\left(p_{8}\right)$.

To encode $x^{n}, y^{n}$, find $m_{0}$ such that $\left(x^{n}, y^{n}\right) \in \mathcal{B}_{0}\left(m_{0}\right)$ and find the index $m_{1}$. Directly encode $y^{n}$ into $m_{2}$.

Decoder 1 receives $m_{0}, m_{1}$ and output $x^{n}$ where $\left(x^{n}, y^{n}\right) \in \mathcal{B}_{0}\left(m_{0}\right)$ with index $m_{1}$. Decoder 3 receives $m_{0}, y^{n}$ and output the unique $\hat{x}^{n}$ such that $\left(\hat{x}^{n}, y^{n}\right) \in \mathcal{B}_{0}\left(m_{0}\right)$. The probability of error vanishes if $H(Y)>H(X, Y)+\epsilon / 2-R_{0}$, which is guaranteed by the definition of $R\left(p_{8}\right)$. Decoder 4 receives $m_{0}, x^{n}$ and output the unique $\hat{y}^{n}$ such that $\left(x^{n}, \hat{y}^{n}\right) \in \mathcal{B}_{0}\left(m_{0}\right)$. The probability of error vanishes if $H(X)>H(X, Y)+\epsilon / 2-R_{0}$, which follows from the definition of $R\left(p_{8}\right)$ and $H(X) \geq H(Y)$.

Hence we have proved that for any point $v \in \mathscr{I}_{X Y}$ and

$$
w \in \mathscr{I}(v)=\left(\left(-\infty, v_{X}\right] \times\left(-\infty, v_{Y}\right] \times\left[v_{X Y}, \infty\right)\right) \cap \mathscr{I}_{X Y}^{\mathrm{o}},
$$

the rate tuple $R(w)$ is achievable. In other words, the region

$$
R\left(\left(\mathscr{I}_{X Y}+(-\infty, 0] \times(-\infty, 0] \times[0, \infty)\right) \cap \mathscr{I}_{X Y}^{\mathrm{o}}\right)+[0, \infty)^{5}
$$


is achievable. The region can be written as

$$
\begin{aligned}
w_{X Y} & \geq I(X, Y ; U), \\
w_{X} & \leq I(X ; U), \\
w_{Y} & \leq I(Y ; U), \\
w_{X} & \geq 0, \\
w_{Y} & \geq 0, \\
w_{X Y}-w_{Y} & \leq H(X \mid Y), \\
w_{X Y}-w_{X} & \leq H(Y \mid X), \\
R_{0} & \geq w_{X Y}+\epsilon, \\
R_{1} & \geq H(X)-w_{X}+\epsilon, \\
R_{2} & \geq H(Y)-w_{Y}+\epsilon, \\
R_{3} & \geq H(X \mid Y)-w_{X Y}+w_{Y}+\epsilon, \\
R_{4} & \geq H(Y \mid X)-w_{X Y}+w_{X}+\epsilon
\end{aligned}
$$

for some $U, w_{X}, w_{Y}, w_{X Y}$. The final rate region can be obtained by eliminating $w_{X}, w_{Y}, w_{X Y}$ using Fourier-Motzkin elimination.

We then prove the converse. Since decoder 3 observes $M_{0}, M_{3}, Y^{n}$ and has to recover $X^{n}$ with vanishing error probability, $R_{0}+R_{3} \geq H(X \mid Y)$. Similarly $R_{0}+R_{4} \geq H(Y \mid X)$. Note that decoders 2 and 3 together can recover $X^{n}, Y^{n}$ with vanishing error probability (decoder 3 uses the output of decoder 2 as the side information), and hence $R_{0}+R_{2}+R_{3} \geq H(X, Y)$. Similarly $R_{0}+R_{1}+R_{4} \geq H(X, Y)$.

Let $U_{i}=\left(M_{0}, X^{i-1}, Y^{i-1}\right)$. Using the same arguments in the proof of Theorem 1 we have $R_{0} \geq I(X, Y ; U), R_{1} \geq$ $H(X \mid U), R_{2} \geq H(Y \mid U)$.

$$
\begin{aligned}
n R_{3} & \geq H\left(M_{3} \mid M_{0}\right) \\
& \geq I\left(X^{n} ; M_{3} \mid M_{0}\right) \\
& =H\left(X^{n} \mid M_{0}\right)-H\left(X^{n} \mid M_{0}, M_{3}\right) \\
& =\sum_{i=1}^{n} H\left(X_{i} \mid M_{0}, X^{i-1}\right)-H\left(X^{n} \mid M_{0}, M_{3}\right) \\
& \geq \sum_{i=1}^{n} H\left(X_{i} \mid M_{0}, X^{i-1}, Y^{i-1}\right)-H\left(Y^{n}\right)-H\left(X^{n} \mid M_{0}, M_{3}, Y^{n}\right) \\
& \geq \sum_{i=1}^{n} H\left(X_{i} \mid U_{i}\right)-H\left(Y^{n}\right)-o(n),
\end{aligned}
$$

where the last inequality is due to Fano's inequality. Similarly $n R_{4} \geq \sum_{i} H\left(Y_{i} \mid U_{i}\right)-H\left(X^{n}\right)-o(n)$.

$$
\begin{aligned}
& n\left(R_{2}+R_{3}\right) \\
& \geq H\left(M_{2}, M_{3} \mid M_{0}\right) \\
& \geq I\left(X^{n} ; M_{2}, M_{3} \mid M_{0}\right) \\
& =H\left(X^{n} \mid M_{0}\right)-H\left(X^{n} \mid M_{0}, M_{2}, M_{3}\right) \\
& =\sum_{i=1}^{n} H\left(X_{i} \mid M_{0}, X^{i-1}\right)-H\left(X^{n} \mid M_{0}, M_{2}, M_{3}\right) \\
& \geq \sum_{i=1}^{n} H\left(X_{i} \mid M_{0}, X^{i-1}, Y^{i-1}\right)-H\left(Y^{n} \mid M_{0}, M_{2}, M_{3}\right)-H\left(X^{n} \mid M_{0}, M_{2}, M_{3}, Y^{n}\right) \\
& \geq \sum_{i=1}^{n} H\left(X_{i} \mid U_{i}\right)-o(n),
\end{aligned}
$$

where the last inequality follows by Fano's inequality. Similarly $n\left(R_{1}+R_{4}\right) \geq \sum_{i} H\left(Y_{i} \mid U_{i}\right)-o(n)$. Hence the point $\left(R_{0}+\right.$ $\left.\epsilon, \ldots, R_{4}+\epsilon\right)$ is in the convex hull of $\mathscr{R}^{\prime}$ for any $\epsilon>0$. We have seen in the achievability proof that (for $\epsilon=0$ )

$$
\mathscr{R}^{\prime}=R\left(\left(\mathscr{I}_{X Y}+(-\infty, 0] \times(-\infty, 0] \times[0, \infty)\right) \cap \mathscr{I}_{X Y}^{\mathrm{o}}\right)+[0, \infty)^{5}
$$

is the increasing hull of an affine transformation of a convex set. Therefore $\mathscr{R}^{\prime}$ is convex. 


\section{E. Proof of Proposition 4}

1) Since the Gray-Wyner region tensorizes, $\operatorname{cl}\left(\mathscr{I}_{X Y}^{\infty}\right) \subseteq\left(\mathscr{I}_{X Y}+(-\infty, 0] \times(-\infty, 0] \times[0, \infty)\right) \cap \mathscr{I}_{X Y}^{\mathrm{o}}$. To prove the other direction, let $w \in\left(\mathscr{I}_{X Y}+(-\infty, 0] \times(-\infty, 0] \times[0, \infty)\right) \cap \mathscr{I}_{X Y}^{\mathrm{o}}$, then by Theorem 2 the following rate tuple is achievable

$$
\begin{aligned}
& R_{0}(w)=w_{X Y}+\epsilon \\
& R_{1}(w)=H(X)-w_{X}+\epsilon \\
& R_{2}(w)=H(Y)-w_{Y}+\epsilon \\
& R_{3}(w)=H(X \mid Y)-w_{X Y}+w_{Y}+\epsilon \\
& R_{4}(w)=H(Y \mid X)-w_{X Y}+w_{X}+\epsilon
\end{aligned}
$$

i.e. for the source $X^{l}, Y^{l}$, the probability of error $P_{e}(l) \rightarrow 0$ as $l \rightarrow \infty$. Apply this scheme $n$ times on the source $X^{n l}, Y^{n l}$. This can be considered as a causal scheme on the source sequence $\left(X_{1}^{l}, Y_{1}^{l}\right),\left(X_{l+1}^{2 l}, Y_{l+1}^{2 l}\right), \ldots,\left(X_{(n-1) l+1}^{n l}, Y_{(n-1) l+1}^{n l}\right)$ with rate tuple $l R(w)$ and symbol error probability $P_{e}(l)$. Hence by (9) and (10) in the proof of Theorem 1

$$
R(w)+\log (|\mathcal{X}| \cdot|\mathcal{Y}|) P_{e}(l) \cdot \mathbf{1} \in(1 / l) \mathscr{R}\left(X^{l} ; Y^{l}\right)
$$

Let $\epsilon^{\prime}=\epsilon+\log (|\mathcal{X}| \cdot|\mathcal{Y}|) P_{e}(l)$. Since

$$
\begin{aligned}
& \frac{1}{l} \mathscr{R}\left(X^{l} ; Y^{l}\right)=\bigcup_{v \in(1 / l) \mathscr{I}_{X^{l} Y^{l}}}\left.v_{X Y}, \infty\right) \times\left[H(X)-v_{X}, \infty\right) \times\left[H(Y)-v_{Y}, \infty\right) \\
& \times\left[H(X \mid Y)-v_{X Y}+v_{Y}, \infty\right) \times\left[H(Y \mid X)-v_{X Y}+v_{X}, \infty\right),
\end{aligned}
$$

there exists $v \in(1 / l) \mathscr{I}_{X^{l} Y^{l}} \subseteq \mathscr{I}_{X Y}^{\infty}$ such that $v_{X Y} \leq w_{X Y}+\epsilon^{\prime}, H(X)-v_{X} \leq H(X)-w_{X}+\epsilon^{\prime}$, and similar for the other 3 dimensions, which implies $\|v-w\|_{\infty} \leq 2 \epsilon^{\prime}$. The result follows from taking $l \rightarrow \infty, \epsilon \rightarrow 0$.

To show

$$
\begin{aligned}
& \left(\mathscr{I}_{X Y}+(-\infty, 0] \times(-\infty, 0] \times[0, \infty)\right) \cap \mathscr{I}_{X Y}^{\mathrm{o}} \\
& =\left(\mathscr{I}_{X Y}+\{(t, t, t): t \leq 0\}\right) \cap([0, \infty) \times[0, \infty) \times \mathbb{R}),
\end{aligned}
$$

note that they are both equal to the union of the convex hulls of $\left\{v, p_{1}, \ldots, p_{8}\right\}$ for $v \in \mathscr{I}_{X Y}$ (as in the proof of Theorem 2).

2) The equivalence between $\operatorname{cl}\left(\mathscr{I}_{X Y}^{\infty}\right)$ and $\mathscr{R}^{\prime}$ is proved in the Fourier-Motzkin elimination step in the proof of Theorem 2

3) By Proposition 2

$$
\begin{aligned}
\mathscr{R}_{\mathrm{GW}} & =\bigcup_{v \in \mathscr{I}_{X Y}}\left\{\left(v_{X Y}, H(X)-v_{X}, H(Y)-v_{Y}\right)\right\}+[0, \infty)^{3} \\
= & \bigcup_{v \in \mathscr{I}_{X Y} \cap \mathscr{I}_{X Y}^{\mathrm{o}}}\left\{\left(v_{X Y}, H(X)-v_{X}, H(Y)-v_{Y}\right)\right\}+[0, \infty)^{3} \\
= & \bigcup_{v \in\left(\mathscr{I}_{X Y}+(-\infty, 0]^{2} \times[0, \infty)\right) \cap \mathscr{I}_{X Y}^{\mathrm{o}}}\left\{\left(v_{X Y}, H(X)-v_{X}, H(Y)-v_{Y}\right)\right\}+[0, \infty)^{3} \\
= & \bigcup_{v \in \operatorname{cl}\left(\mathscr{I}_{X Y}^{\infty}\right)}\left\{\left(v_{X Y}, H(X)-v_{X}, H(Y)-v_{Y}\right)\right\}+[0, \infty)^{3} .
\end{aligned}
$$

For the other direction,

$$
\begin{aligned}
\operatorname{cl}\left(\mathscr{I}_{X Y}^{\infty}\right) & =\left(\mathscr{I}_{X Y}+(-\infty, 0]^{2} \times[0, \infty)\right) \cap \mathscr{I}_{X Y}^{\mathrm{o}} \\
& =\left\{v \in \mathscr{I}_{X Y}^{\mathrm{o}}: v_{X} \leq w_{X}, v_{Y} \leq w_{Y}, v_{X Y} \geq w_{X Y} \text { for some } w \in \mathscr{I}_{X Y}\right\} \\
& =\left\{v \in \mathscr{I}_{X Y}^{\mathrm{o}}: v_{X} \leq I(X ; U), v_{Y} \leq I(Y ; U), v_{X Y} \geq I(X, Y ; U) \text { for some } U\right\} \\
& =\left\{v \in \mathscr{I}_{X Y}^{\mathrm{o}}: H(X)-v_{X} \geq H(X \mid U), H(Y)-v_{Y} \geq H(Y \mid U), v_{X Y} \geq I(X, Y ; U) \text { for some } U\right\} \\
& =\left\{v \in \mathscr{I}_{X Y}^{\mathrm{o}}:\left(v_{X Y}, H(X)-v_{X}, H(Y)-v_{Y}\right) \in \mathscr{R}_{\mathrm{GW}}\right\} .
\end{aligned}
$$

\section{REFERENCES}

[1] R. M. Gray and A. D. Wyner, "Source coding for a simple network," Bell Syst. Tech. J., vol. 53, no. 9, pp. 1681-1721, 1974.

[2] A. D. Wyner, "The common information of two dependent random variables," IEEE Trans. Inf. Theory, vol. 21, no. 2, pp. 163-179, Mar. 1975.

[3] P. Gács and J. Körner, "Common information is far less than mutual information," Probl. Control Inf. Theory, vol. 2, no. 2, pp. 149-162, 1973.

[4] P. Cuff, H. Permuter, and T. M. Cover, "Coordination capacity," IEEE Trans. Info. Theory, vol. 56, no. 9, pp. 4181-4206, 2010.

[5] N. Tishby, F. C. Pereira, and W. Bialek, "The information bottleneck method," arXiv preprint physics/0004057, 2000.

[6] A. D. Wyner, J. K. Wolf, and F. M. J. Willems, "Communicating via a processing broadcast satellite," IEEE Transactions on Information Theory, vol. 48, no. 6, pp. 1243-1249, Jun 2002. 
[7] A. Kimura and T. Uyematsu, "Multiterminal source coding with complementary delivery," in Proc. IEEE Int. Symp. Inf. Theory Appl., Seoul, Korea, October 2006, pp. 189-194.

[8] J. Körner, "Coding of an information source having ambiguous alphabet and the entropy of graphs," in 6th Prague conference on information theory, 1973, pp. 411-425.

[9] A. Makhdoumi, S. Salamatian, N. Fawaz, and M. Medard, "From the information bottleneck to the privacy funnel," in Information Theory Workshop (ITW), 2014 IEEE, Nov 2014, pp. 501-505.

[10] C. T. Li and A. El Gamal, "Strong functional representation lemma and applications to coding theorems," arXiv preprint, 2017. [Online]. Available: http://arxiv.org/abs/1701.02827

[11] W. J. McGill, "Multivariate information transmission," Psychometrika, vol. 19, no. 2, pp. 97-116, 1954. [Online]. Available: http://dx.doi.org/10.1007/BF02289159

[12] T. Weissman and A. El Gamal, "Source coding with limited-look-ahead side information at the decoder," IEEE Trans. Inf. Theory, vol. 52, no. 12, pp. 5218-5239, Dec. 2006.

[13] P. Cuff, "Distributed channel synthesis," IEEE Trans. Info. Theory, vol. 59, no. 11, pp. 7071-7096, 2013.

[14] C. H. Bennett, I. Devetak, A. W. Harrow, P. W. Shor, and A. Winter, "The quantum reverse shannon theorem and resource tradeoffs for simulating quantum channels," IEEE Trans. Info. Theory, vol. 60, no. 5, pp. 2926-2959, May 2014.

[15] A. D. Wyner, "A theorem on the entropy of certain binary sequences and applications-II," IEEE Trans. Inf. Theory, vol. 19, no. 6, pp. 772-777, 1973.

[16] R. Ahlswede and J. Körner, "Source coding with side information and a converse for degraded broadcast channels," IEEE Trans. Inf. Theory, vol. 21, no. 6, pp. 629-637, 1975 .

[17] A. D. Wyner, "On source coding with side information at the decoder," IEEE Trans. Inf. Theory, vol. 21, no. 3, pp. 294-300, 1975.

[18] R. Timo and B. N. Vellambi, "Two lossy source coding problems with causal side-information," in 2009 IEEE International Symposium on Information Theory, June 2009, pp. 1040-1044.

[19] A. D. Wyner and J. Ziv, "The rate-distortion function for source coding with side information at the decoder," IEEE Trans. Inf. Theory, vol. 22, no. 1, pp. $1-10,1976$.

[20] C. Heegard and T. Berger, "Rate distortion when side information may be absent," IEEE Transactions on Information Theory, vol. 31, no. 6, pp. 727-734, Nov 1985.

[21] Y. Steinberg and N. Merhav, "On successive refinement for the Wyner-Ziv problem," in International Symposium onInformation Theory, 2004. ISIT 2004. Proceedings., 2004, pp. 364-364.

[22] C. Tian and S. N. Diggavi, "Side-information scalable source coding," IEEE Transactions on Information Theory, vol. 54, no. 12, pp. 5591-5608, Dec 2008.

[23] V. M. Prabhakaran and M. M. Prabhakaran, "Assisted common information with an application to secure two-party sampling," IEEE Transactions on Information Theory, vol. 60, no. 6, pp. 3413-3434, June 2014.

[24] M. M. Prabhakaran and V. M. Prabhakaran, "Tension bounds for information complexity," arXiv preprint arXiv:1408.6285, 2014.

[25] H. S. Witsenhausen, "On sequences of pairs of dependent random variables," SIAM Journal on Applied Mathematics, vol. 28, no. 1, pp. 100-113, 1975.

[26] N. Alon and A. Orlitsky, "Source coding and graph entropies," IEEE Transactions on Information Theory, vol. 42, no. 5, pp. 1329-1339, Sep 1996.

[27] S. Wolf and J. Wullschleger, "New monotones and lower bounds in unconditional two-party computation," IEEE Transactions on Information Theory, vol. 54, no. 6, pp. 2792-2797, June 2008.

[28] S. Kamath and V. Anantharam, "A new dual to the Gács-Körner common information defined via the Gray-Wyner system," in Communication, Control, and Computing (Allerton), 2010 48th Annual Allerton Conference on, Sept 2010, pp. 1340-1346.

[29] S. Asoodeh, F. Alajaji, and T. Linder, "Notes on information-theoretic privacy," in Communication, Control, and Computing (Allerton), 2014 52nd Annual Allerton Conference on, Sept 2014, pp. 1272-1278.

[30] P. K. Banerjee and V. Griffith, "Synergy, redundancy and common information," arXiv preprint arXiv:1509.03706, 2015.

[31] R. Ahlswede and I. Csiszar, "Common randomness in information theory and cryptography. II. CR capacity," IEEE Transactions on Information Theory, vol. 44, no. 1, pp. 225-240, Jan 1998.

[32] H. Witsenhausen and A. Wyner, "A conditional entropy bound for a pair of discrete random variables," IEEE Transactions on Information Theory, vol. 21, no. 5, pp. 493-501, Sep 1975 .

[33] V. Anantharam, A. A. Gohari, S. Kamath, and C. Nair, "On maximal correlation, hypercontractivity, and the data processing inequality studied by erkip and cover," CoRR, vol. abs/1304.6133, 2013. [Online]. Available: http://arxiv.org/abs/1304.6133

[34] R. Ahlswede and P. Gács, "Spreading of sets in product spaces and hypercontraction of the Markov operator," The annals of probability, pp. 925-939, 1976.

[35] F. P. Calmon, A. Makhdoumi, and M. Medard, "Fundamental limits of perfect privacy," in 2015 IEEE International Symposium on Information Theory (ISIT), June 2015, pp. 1796-1800.

[36] P. Harsha, R. Jain, D. McAllester, and J. Radhakrishnan, "The communication complexity of correlation," IEEE Trans. Info. Theory, vol. 56, no. 1, pp. 438-449, Jan 2010

[37] D. E. Knuth and A. C. Yao, "The complexity of nonuniform random number generation," Algorithms and complexity: new directions and recent results, pp. 357-428, 1976.

[38] H. G. Eggleston, Convexity. Cambridge: Cambridge University Press, 1958.

[39] R. T. Rockafellar, Convex Analysis. Princeton, NJ: Princeton University Press, 1970.

[40] A. El Gamal and Y.-H. Kim, Network information theory. Cambridge university press, 2011. 